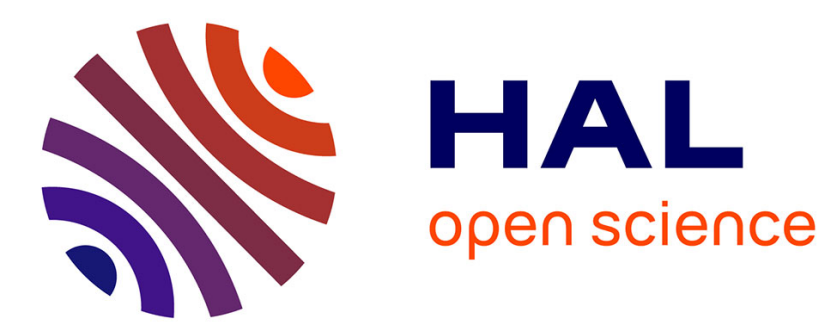

\title{
Ageing and Degradation of Multiphase Polymer Systems
} Xavier Colin, G. Teyssedre, Magali Fois

\section{To cite this version:}

Xavier Colin, G. Teyssedre, Magali Fois. Ageing and Degradation of Multiphase Polymer Systems. Abderrahim Boudenne; Laurent Ibos; Yves Candau; Sabu Thomas. Handbook of Multiphase Polymer Systems, Wiley, pp.797-841, 2011, 978-0-4707-1420-1. 10.1002/9781119972020.ch21 . hal-02618425

\section{HAL Id: hal-02618425 \\ https://hal.science/hal-02618425}

Submitted on 25 May 2020

HAL is a multi-disciplinary open access archive for the deposit and dissemination of scientific research documents, whether they are published or not. The documents may come from teaching and research institutions in France or abroad, or from public or private research centers.
L'archive ouverte pluridisciplinaire HAL, est destinée au dépôt et à la diffusion de documents scientifiques de niveau recherche, publiés ou non, émanant des établissements d'enseignement et de recherche français ou étrangers, des laboratoires publics ou privés. 


\title{
Ageing and Degradation of Multiphase Polymer Systems
}

\author{
Xavier Colin \\ PIMM (UMR 8006), Arts et Métiers ParisTech, Paris, France \\ Gilbert Teyssedre \\ Laplace, Université Paul Sabatier, Toulouse, France \\ Magali Fois \\ Université Paris-Est, CERTES EA 3481 - Centre d'Etude et de Recherche en Thermique, Environnement et \\ Systèmes, Créteil, France
}

\subsection{Introduction}

\subsubsection{Issues Associated with Material Ageing}

Ageing can be defined as a slow and irreversible variation as a function of time (in use conditions) of a material structure, morphology or composition leading to a detrimental change in its use properties. The cause of this change can be the own material instability or its interaction with the environment of exposure. The definition so given is that viewed from an application point of view. There are issues associated with this definition that deserve to be mentioned. First, there are ageing mechanisms, essentially of a physical nature as detailed below, that are not irreversible in nature (e.g. crystallinity change, structural recovery, water uptake without loss of the integrity of the chemical structure, etc.), but may lead to a change in the use properties of these materials. The reversibility is in principle achievable by, e.g. thermal treatment or drying. However, this is not necessarily compatible with the use of materials as pieces, or the material will evolve again anyway when exposed to use environmental stresses. Second, irreversible material evolution in itself does not necessarily imply a detrimental change of use properties. It can even result in an improvement of properties. This represents indeed a marked difficulty when attempting to define so-called 'ageing markers' for materials, i.e. material properties to be monitored for health monitoring purpose: the marker must be 
sensitive enough so as to provide the early signs of material evolution but, at the same time, there should be a correlation between the evolution of the marker and the changes in use properties.

In the following, we shall consider ageing from the point of view of molecular, macromolecular or microscopic changes induced on the polymer, with room given on the impact on use properties. A large reference to the impact of various ageing mechanisms on mechanical properties will be made, in part because of the area of expertise of the authorship, and most importantly because of the more advanced knowledge and earlier consideration of the relationships between mechanistic ageing processes and macroscopic mechanical properties. This is even more evident when dealing with polymer-based composite materials which have emerged for the huge improvement of mechanical properties brought about in respect to the matrix alone, and which do still represent the essential part of the quantity of material being produced and a significant part of the research being carried out on the topic. Any polymer or composite piece has a mechanical role, and even in demanding applications such as electrical insulation, it can be more advisable to use composites instead of polymers alone for mechanical or thermal criteria (along with economics), even though it can be significantly detrimental to the electrical performances. In another respect, the policy of switching to all-composite epoxy-carbon aircrafts has been guided by mechanical engineering and the perspective offered in weight reduction with, to our eyes, little consideration of the consequences it may have on electrical networks onboard and on lightning issues.

The challenges when dealing with ageing of materials are multiple, going from mechanistic processes, to materials selection and to design. In terms of area of expertise, it implies gathering people with skills ranging from macromolecular chemistry to engineering resorting to the various area of application of materials. The main tasks that are currently addressed, irrespective of materials considered and targeted application, are as follows:

(a) understand how the material is evolving

(b) find the ageing markers relevant to the expected service properties

(c) define 'end-of-life' criteria and forecast material evolution (life estimation)

(d) find ways to prevent material ageing.

\subsubsection{Classification of Ageing Types}

There is a wide variety of polymer ageing types. Each one is a complex phenomenon, but decomposable into a set of elementary mechanisms that can be interactive: chemical reactions, macromolecular motions, molecular species transport into the polymer, crystallinity change, etc. Unfortunately, there is no entirely satisfactory method for their classification, because the most rigorous criteria, in theory, are often open to criticism in practice. The simplest and most agreed method consists in distinguishing two main ageing families according to the nature of involved mechanisms:

- chemical ageing is responsible for a variation of the macromolecule chemical structure

- physical ageing affects the macromolecule conformation, the material morphology or composition, without altering the macromolecule chemical structure.

Then, the different ageing types, defined in terms of the main ageing driving environmental stress, involve one of the two families of mechanisms defined above:

- Thermal ageing is initiated by material's own instability. Its kinetics essentially depends on temperature and atmosphere composition. It can be a chemical or a physical ageing, or a combination of them.

- Humid ageing is due to a chemical or a physical interaction of polymer with water, the latter being either in a liquid or a vapor state. 
- Photo-chemical ageing results from polymer interaction with light rays (in general, UV solar radiations). Atmospheric oxygen plays, in general, an important role (photo-oxidation).

- Radio-chemical ageing is due to the polymer interaction with ionizing ( $\gamma, \beta, \alpha$, neutrons, etc.) rays. Atmospheric oxygen can play an important role, especially at very low dose rate (radio-oxidation).

- Electrical ageing due to the interaction of electrical charges with the polymer or to a direct field effect on it.

- Mechanical ageing through, e.g. fatigue exerted on the polymer which can induce chain breaking along with morphological changes within the material.

- Biochemical ageing is due to the polymer interaction with living organisms (bacteria, molds, etc.).

Ageing is a difficult problem to study in practice, because it usually proceeds slowly in (soft) service conditions of materials and lifetime reaches typically several dozens of years. It is therefore not advisable to test and qualify materials for a given application on a natural ageing basis. Accelerated ageing tests are then performed in laboratories in order to elaborate a relatively simple kinetic model describing the material behavior changes and predicting the lifetime from a conventional end-of-life criterion in a reasonable time scale. However, the pertinences of the choice of accelerated ageing conditions, the mathematical form of kinetic model and the end-of-life criterion are rarely discussed and never demonstrated. The use of such an empirical method and, thus, the reliability of its predictions by extrapolation, are highly questionable.

In contrast, one can define a nonempirical method for lifetime prediction which would involve at least four elementary steps:

(a) identification of elementary ageing mechanisms, and their eventual couplings, through the analytical observations reported in the literature

(b) elaboration of a general kinetic model from the previously determined elementary mechanisms

(c) determination of model parameters from accelerated ageing tests

(d) extrapolation of the considered use properties to (soft) use conditions and lifetime prediction from a critical structural state, using polymer physics.

All these steps are very important. Ignoring just one of them can have dramatic consequences on lifetime prediction by extrapolation. The nonempirical method has been applied with success in various industrial applications, for instance, quite recently, in the case of sulphur vulcanized polyisoprene (SVPIP) seals for aerospace applications [1] or carbon black filled polyethylene (CBPE) pipes for the transport of drinking water [2].

The present chapter is devoted to the first step of the nonempirical method. The main polymer (physical and chemical) ageing types, susceptible to take place in practice, will be presented. Particular attention will be paid to embrittlement mechanisms and their corresponding kinetic laws, in order to give to the reader the necessary theoretical bases for a future kinetic modeling.

\subsection{Physical Ageing}

Physical ageing can result from the spatial reorganization of macromolecules (relaxation of enthalpy, volume, orientation or stress, crystallization, etc.), transport phenomena (penetration of a solvent, migration of an additive) and superficial phenomena (cracking in a tensioactive medium). 


\subsubsection{Ageing Induced by Structural Reorganization}

Liquid-to-glass transition and crystallization are both responsible for polymer solidification in the end of a processing operation. Since they are kinetic phenomena, they lead to an out-of-equilibrium thermodynamic state:

- glassy polymers present an excess of unstable conformations and free volume

- semicrystalline polymers are not totally crystallized, their melting point being largely lower (usually some dozens of degrees) than the equilibrium value.

If, in their use conditions, polymers of either type are subjected to a residual molecular mobility ( $\beta$ motions in glassy polymers, $\alpha$ motions in the rubbery amorphous phase of semicrystalline polymers), they undergo molecular reorganization towards the thermodynamic equilibrium. The main consequences of this ageing type, inappropriately called 'physical ageing' since there are other types of physical ageing, best defined as structural relaxation are:

- a compaction of macromolecules and a loss in enthalpy

- an increase in yield stress

- a decrease in creep compliance

- the variation against time of the previously quoted quantities is auto-retarded, but can proceed for historic durations.

The variation against time of creep compliance $J(t)$ has been the subject of many literature works, in particular in the case of organic glasses [3]. As an example, the general shape of creep curves of samples aged during three different durations: $t_{a}, 10 \times t_{a}$ and $100 \times t_{a}$, is presented in Figure 21.1.

The time-shift in creep curves, defined as $a_{c}=\log \left(t_{1 c} / t_{2 c}\right)$, corresponding to a variation in ageing time $a_{a}=\log \left(t_{1 a} / t_{2 a}\right)$ is such that $a_{a} / a_{c} \approx 1$. In other words, one decade increase in ageing time leads to about one decade increase in the creep characteristic time. It is a general trend of the physical ageing by structural relaxation observed for polymers or molecular organic materials (glucose), as well as for granular solids like sand, or emulsions.

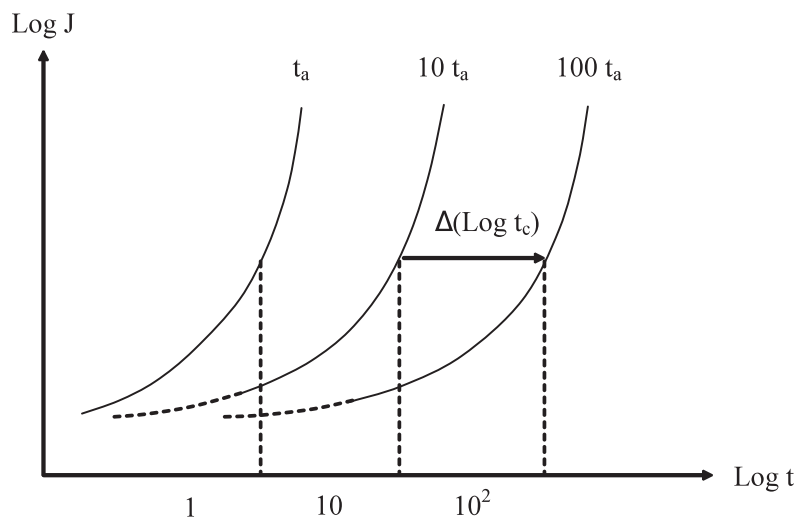

Figure 21.1 Creep curves (in a log-log scale) of samples aged during $t_{a}, 10 \times t_{a}$ and $100 \times t_{a}$ in their glassy state. 


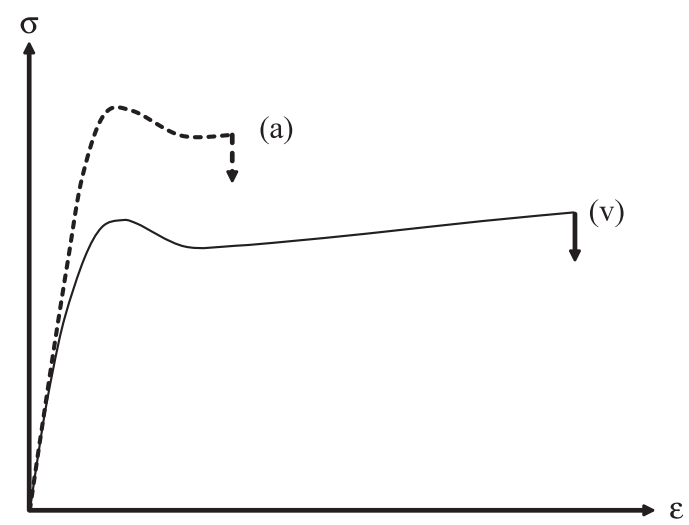

Figure 21.2 Shape of stress $(\sigma)$ vs. strain $(\varepsilon)$ curves before $(v)$ and after (a) structural relaxation. Vertical arrows symbolize sample failure.

The decrease in creep compliance (i.e. increase in Young's modulus), as well as the increase in yield stress, can be viewed as advantages for many applications; however, these changes are counterbalanced by a catastrophic decrease in ductility / toughness, as schematized in Figure 21.2. The consequence of structural relaxation can be understood as an increase in the fragility of the material.

The increase in yield stress (typically, up to $30 \%$ of its initial value for a polycarbonate (PC)), the loss in ductility without any variation of the molecular weight distribution, plus the appearance of an endothermic peak close to the glass transition temperature $\left(T_{g}\right)$ (Figure 21.3) in differential scanning calorimetry (DSC) thermograms allows to unambiguously identify the structural relaxation from any another type of physical ageing in the case of amorphous glassy polymers.

Perhaps one of the most dramatic changes in physical properties of amorphous polymers induced by physical ageing is that associated with gas transport properties [4] of polymer membranes with a diffusion coefficient decreasing by nearly one decade. This is a direct effect of the decrease in free volume induced by structural relaxation.

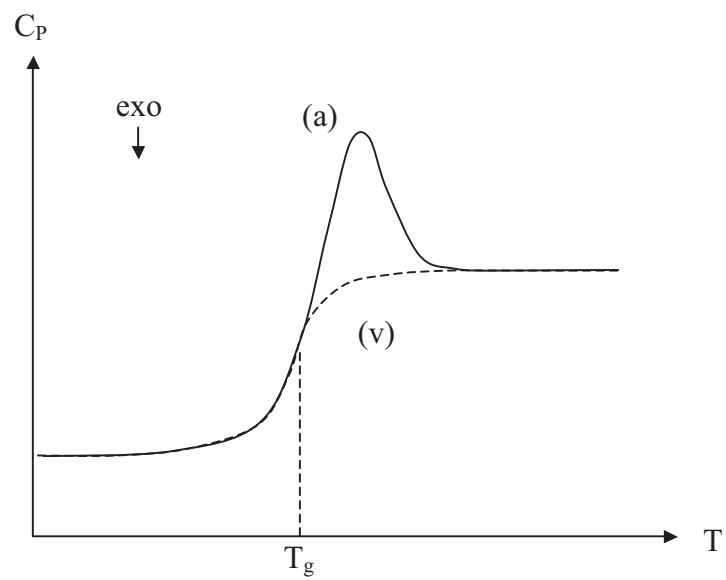

Figure 21.3 Shape of DSC thermograms around the glass transition temperature ( $\left.T_{g}\right)$ before $(v)$ and after (a) structural relaxation. 


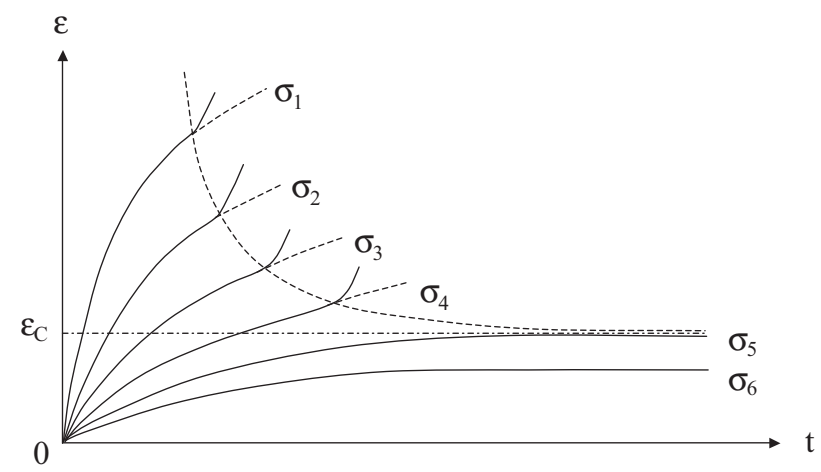

Figure 21.4 Shape of creep curves (time variation of strain) for different stress levels $\left(\sigma_{1}>\sigma_{2}>\sigma_{3} \ldots\right.$ etc) in the presence of a solvent. Black points indicate the appearance of damage. Their envelope follows a horizontal asymptote for $\varepsilon=\varepsilon_{c}$.

\subsubsection{Ageing Induced by Solvent Absorption}

Solvents plasticize polymers and thus, lead to a decrease in $T_{g}$ and yield stress (if the polymer is initially ductile).

However, the most significant effects, in practice, are observed when the polymer is subjected to a mechanical loading. In this case, plasticization favors damage, in particular by crazing.

As an example, let us consider a creep test during which damage is detected by an optical technique. The resulting behavior can be schematized in Figure 21.4.

One can see that there is a critical strain $\varepsilon_{C}$ below which the material does not damage. The value of $\varepsilon_{C}$ is a function of the polymer-solvent couple. In the simplest cases (moderately polar polymers such as poly(2,6-dimethyl oxyphenylene) (PPO) [5]), $\varepsilon_{C}$ depends on the parameter of solvent solubility as shown in Figure 21.5. The dashed zone corresponds to values of $\varepsilon_{C}$ determined in air. In the case of polar polymers

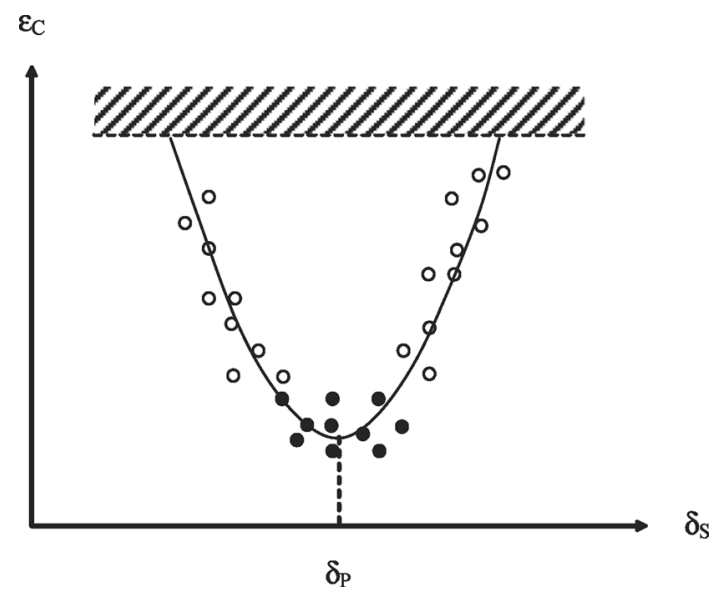

Figure 21.5 Shape of the variation, against parameter of solvent solubility $\left(\delta_{S}\right)$, of critical strain $\left(\varepsilon_{C}\right)$ for PPO [5]. Minimum of curve corresponds to: $\delta_{S}=\delta_{P}, \delta_{P}$ being the parameter of polymer solubility. (०) crazes; (•) open cracks. 
(for instance, poly(methyl methacrylate) (PMMA)), the behavior can be more complex: the curve $\varepsilon_{C}=f\left(\delta_{S}\right)$ can exhibit several minima.

Some vapors, like water or carbon dioxide, play an important role in solvent-induced ageing. Moreover, plasticizers can migrate from one polymer to another and thus generate damage under mechanical loading. In the case of relatively complex pieces, in the presence of vapors, localized damage can be enhanced by the presence of residual stresses due to the processing conditions.

The penetration of solvents into a polymer leads to swelling and can also lead to stress gradients induced by hindered swelling during the transient regime of diffusion. Problems of organic matrix composite damage induced by water diffusion have been reported in the literature over the half past century, in particular in aeronautics $[6,7,8]$.

\subsubsection{Ageing Induced by Additive Migration}

Most technical polymers contain additives like antioxidants, plasticizers or processing agents that do not form chemical bonds with macromolecules. These molecules can migrate within the polymer at a speed depending on the size of the molecules, their solubility and diffusivity within the host polymer among other parameters. Plasticizers (of poly(vinyl chloride) (PVC), for instance) are well-known for their propensity to migrate into any types of polymers. The consequence is an embrittlement of the material. We present first the limiting steps in additive migration.

A polymer-additive mixture is out of thermodynamic equilibrium, since the additive concentration in the environment being equal to zero, there is no equality between its chemical potentials in the environment and in the polymer. Additive molecules tend to migrate outside the polymer in order to establish this equality and thus, to reach an equilibrium. This migration is composed of two steps (Figure 21.6):

1 The first step corresponds to the passing of some additive molecules into a medium of molecules close to the polymer surface, that is to say the crossing of the polymer-external medium interface. In a gaseous medium (for instance, atmosphere), the additive evaporation controls the kinetics of this step. In a liquid medium, the additive dissolution within the liquid plays the same role. The external medium can be a polymer in contact with the first one, and again it is the additive dissolution which is the controlling step. A consequence of the last example is that polymers tend to exchange their antioxidants.

2 The additive molecule exchange between the polymer and the external medium leads to a concentration gradient in a region adjacent to the surface of the material. This latter is the 'driving force' for the

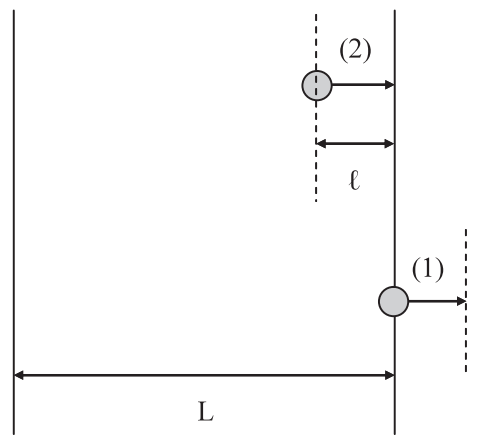

Figure 21.6 Schematization of a two-step-migration of molecular species outside the polymer. $L$ is the sample thickness. 
diffusion of additive molecules from the core towards the sample surface. If diffusion obeys the second Fick's law, the diffusion distance $(\ell)$ is proportional to the square-root of time $\left(t_{d}\right)$, i.e.:

$$
t_{d}=\frac{\ell^{2}}{D}
$$

where $D$ is the diffusion coefficient of additive into the polymer.

Note that we treat here Fickian diffusion processes, which may hold essentially far from $T_{g}$ transition of polymer networks. There are many exceptions to that process and we invite the reader to refer to extensive reviews available in the literature for the treatment of transport of small molecules into polymers [9, 10, 11].

One can see from Eq. (21.1) that $t_{d}$ increases rapidly with the sample thickness. From processes (1) and (2) described above, one can distinguish two distinct kinetic regimes of diffusion, depending on which process is the rate limiting step.

Regime 1: Evaporation (or dissolution) controlled kinetics In the case of thin samples (fibers, films, coatings, etc.) and high additive diffusivity, additive evaporation is the slowest step and thus controls the global migration kinetics. Its concentration $C$ (into the polymer) decreases proportionally with time (see Figure 21.7, left):

$$
\frac{d C}{d t}=-H
$$
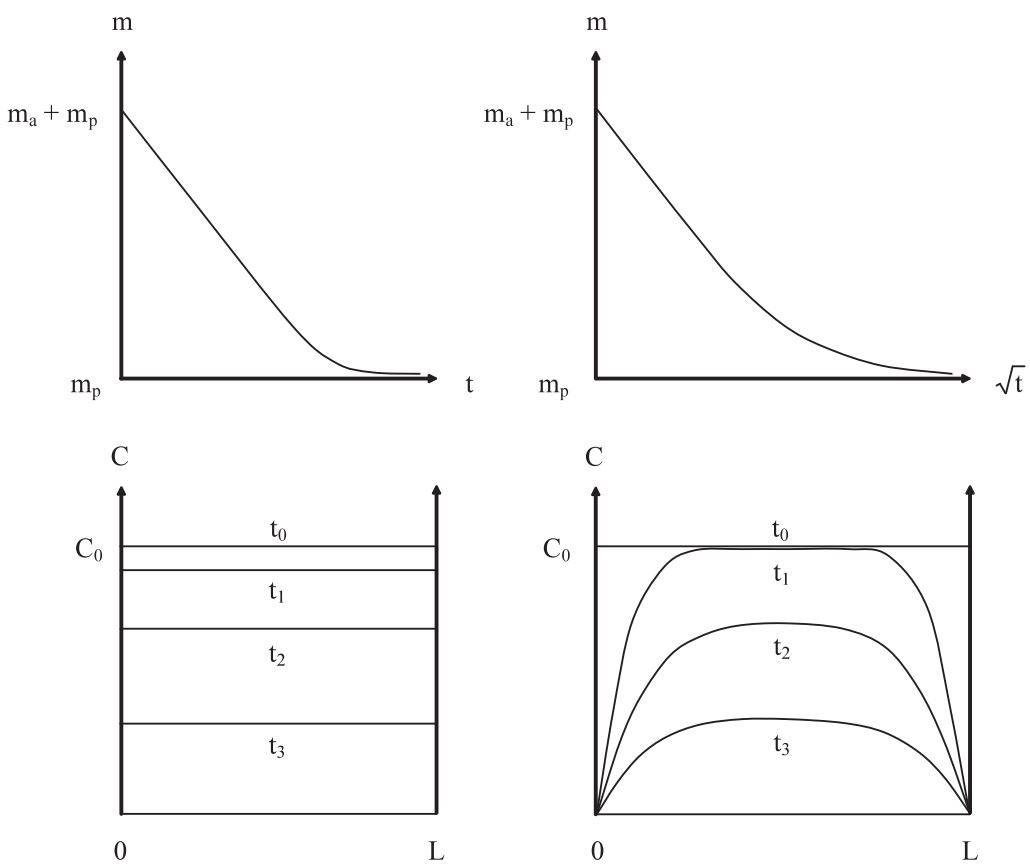

Figure 21.7 Additive migration governed by evaporation (left) and diffusion (right). Top: shape of weight changes as a function of time. Sample weight $(m)$ corresponds to polymer weight $\left(m_{p}\right)$ plus additive weight $\left(m_{a}\right)$; Bottom: distribution, in the sample thickness, of additive concentration ( $C$ ) for different times of exposure: $t_{0}<t_{1}<t_{2}<t_{3}$. The initial concentration is $C=C_{0}\left(a t t=t_{0}\right)$. 
The evaporation rate, $H$, is a decreasing function of the additive molar mass and cohesive energy density.

Regime 2: Diffusion controlled kinetics In the case of relatively thick samples (typically few millimetres thick) and low additive diffusivity, diffusion in the bulk controls the global migration kinetics. In the simplest case, the second Fick's law can be successfully applied. It is found that, in the early period of exposure, the sample weight $m$ decreases proportionally with the square root of time (see Figure 21.7, right):

$$
\frac{\partial m}{\partial t}=D \frac{\partial^{2} m}{\partial z^{2}}
$$

where $z$ is the spatial coordinate in the sample thickness.

Thus, a gradient of additive concentration appears in the sample thickness. Figure 21.7 shows the shape of the time variation of sample mass and concentration profile in the case of evaporation controlled and diffusion controlled additive migration.

If the additive molar mass is relatively high, its evaporation (or, more generally speaking, its crossing of the polymer-medium interface) is slow. Then, its concentration at the sample surface takes an intermediate value between $C_{0}$ and zero. It is necessary to take into account this variation in the boundary conditions, for the resolution of Eq. (21.3).

If the additive concentration is high (as in the case of plasticizers), it modifies the polymer properties. Then, its diffusivity becomes concentration dependent. Complications appear when a phase transition takes place during the additive migration. As an example, in the case of diffusion of plasticizers in PVC, a dramatic increase of $T_{g}$ occurs in regions of low plasticizer concentration. Hence, the $T_{g}$ profile can have the shape illustrated in Figure 21.8. Naturally, the increase in $T_{g}$ at the sample surface will lead to a decrease of the

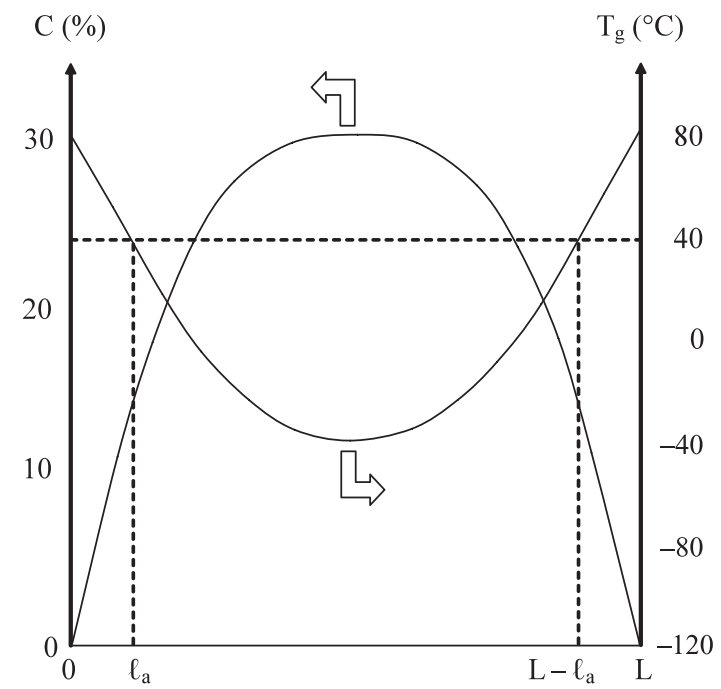

Figure 21.8 Shape of distribution, in the sample thickness, of plasticizer concentration (C expressed in weight fraction) and resulting local glass transition temperature ( $\left.T_{g}\right)$ or a plasticized PVC aged at room temperature [12]. 


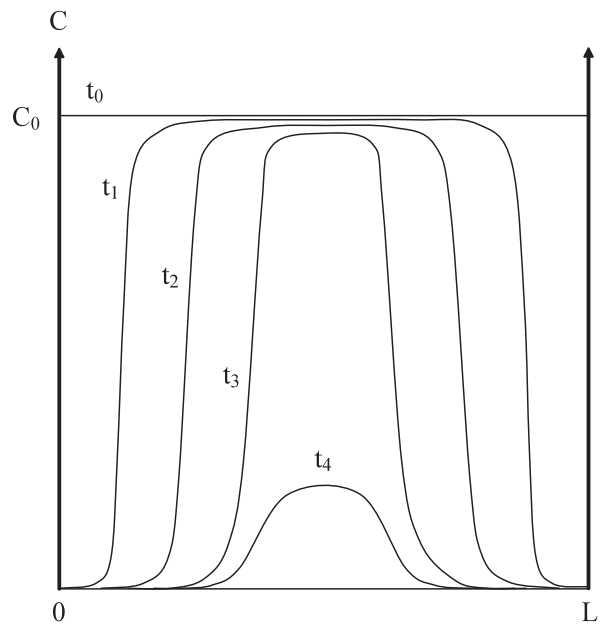

Figure 21.9 Correction of the hypothetic curve (right) presented in Figure 21.7. Real shape of the distribution, in the sample thickness, of plasticizer concentration (C) for different times of exposure: $t_{0}<t_{1}<t_{2}<t_{3}<t_{4}$.

diffusivity in that region, along with, possibly, a change in transport process. The material surface becomes brittle with a 'soft' bulk region.

One can see that, in the bulk of the sample, the polymer remains in a rubbery state. On the contrary, in the superficial layer of thickness $\ell_{a}$, the polymer is in a glassy state. Since the coefficient of plasticizer diffusion varies of at least one order of magnitude on both sides of the glass transition temperature, the real gradient will display rather the shape of Figure 21.9.

In such cases, the diffusion 'front' is very abrupt and the sample weight decreases proportionally with time.

On the mechanical point of view, the loss in additives leads to a loss in the specific properties obtained by the introduction of these latter: long-term durability in the case of antioxidants, flexibility in the case of plasticizers, etc. If the additive concentration is high (as with plasticizers), their loss induces a volume shrinkage (of the order of the weight loss). This shrinkage can generate local stresses and thus lead to cracking.

\subsection{Chemical Ageing}

\subsubsection{General Aspects}

\subsubsection{The Two Main Families of Chemical Ageing}

It is important to distinguish between two types of chemical ageing processes: on the one hand, those leading to change in lateral groups of macromolecules, without affecting the macromolecular skeleton; on the other, those leading also to a change in the macromolecular skeleton. Indeed, in the first case no significant change in the mechanical properties at low conversion ratio of the reaction is produced, whereas the second type induces an important change of mechanical properties, even at low conversion ratio.

Mechanisms Leading to a Change in Lateral Groups Halogenation and sulfonation reactions are susceptible to substitute hydrogen atoms by various groups, but such mechanisms are rarely encountered in use conditions. At the opposite, oxidation reactions are very common. These reactions may affect also the 
macromolecular skeleton but, at this stage, only their consequences on the macromolecule chemical structure will be considered. Examples of lateral groups produced by oxidation are the following:<smiles>CCCCCCC(C)O</smiles>

A hydrocarbon group, moderately polar, is transformed into a polar (ketone) or highly polar (alcohol, acid) oxygenated group. If this change occurs in a polymer initially rich in polar groups, it has limited consequences on physical properties. However, if this change occurs in a polymer initially not very polar (such as polyolefins or hydrocarbon elastomers), it induces a significant variation of all the physical properties depending on the macromolecule chemical structure, in particular: coloring, dielectric permittivity, refractive index, surface energy and wettability.

Some mechanisms lead to the formation of colored species. As an example, in the case of PVC, one of them is the sequential (zip) elimination of hydrogen chloride:

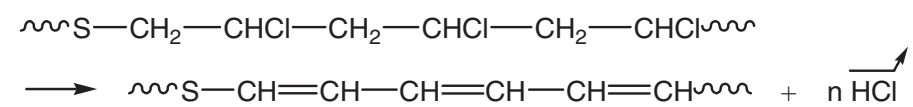

where $\mathrm{S}$ is a structural irregularity (for instance, a tertiary or an allylic chloride formed during polymer synthesis) destabilizing the neighboring monomer unit.

Sequential elimination is favored because allylic chlorides are especially unstable. It leads to the formation of conjugated polyenes:

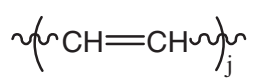

with $j$ ranging typically from 1 to 20 .

The absorption spectra of polyenes in the UV-visible range are schematised in Figure 21.10 [13]. As a general rule, for $j \geq 6$, polyenes absorb in the UV-visible range, and the absorption spectrum is shifted towards long wavelength values as the number of conjugated double bonds increases. The polymer appears yellow for $j \leq 8$, violet-purple for $j=12-15$, and brown to black for higher values of $j$.

Since the resulting chemical species present a very high coefficient of molar absorptivity, of the order of $10^{5} 1 . \mathrm{mol}^{-1} . \mathrm{cm}^{-1}$, coloration appears at low, and sometimes at very low, conversion ratio of the reaction.

In the case of aromatic polymers, oxidation leads to chromophoric species, in particular quinoleic species absorbing in the near UV and violet range:

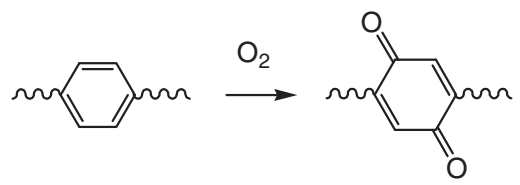

Thus, these polymers (polystyrene (PS), polycarbonate (PC), poly(ethylene terephthalate) (PET), styrene crosslinked polyesters (UP), epoxy, etc) undergo yellowing during exposure to oxidation conditions.

Oxidation of saturated hydrocarbon polymers (for instance, polyethylene (PE) and polypropylene (PP)) can also lead to colored species if the material contains initially traces of metallic impurities susceptible to form colored complexes with some oxidation products such as carbonyl groups. 


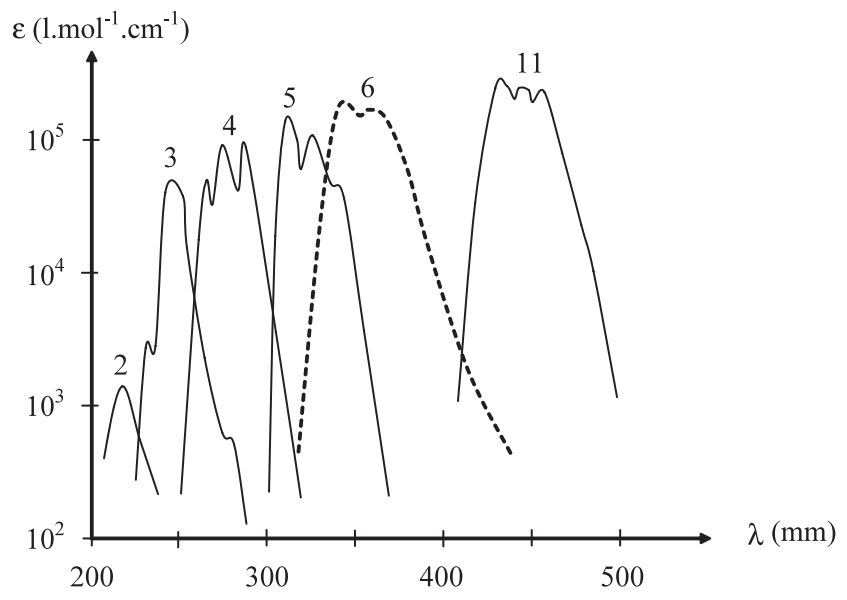

Figure 21.10 Shape of UV-visible spectra for conjugated polyenes as a function of the conjugation degree $j$ indicated in the figure [13]. The absorption wavelength peak $(\lambda)$ and molar absorptivity $(\varepsilon)$ increase with conjugation length.

Finally, some stabilisation mechanisms, in particular with aromatic amines or hindered phenols, are susceptible to leading also to highly-colored species (due to antioxidant reaction). However, these latter can be easily distinguished from colored species belonging to macromolecules, since they are extractible by using an adequate solvent.

Mechanisms Leading to a Change of Macromolecular Skeleton One can distinguish two distinct types of ageing induced at the level of the macromolecular skeleton: chain scission and crosslinking (Figure 21.11). Of course, both types can take place simultaneously.

If $S$ and $X$ are respectively the numbers of moles of chain scission and chain welding per mass unit, one can write [14]:

$$
\begin{aligned}
& \frac{1}{M_{n}}-\frac{1}{M_{n 0}}=S-X \\
& \frac{1}{M_{W}}-\frac{1}{M_{W 0}}=\frac{S}{2}-2 X
\end{aligned}
$$

- Random chain scission:

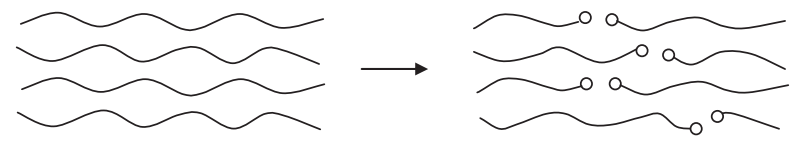

- Chain welding (crosslinking):

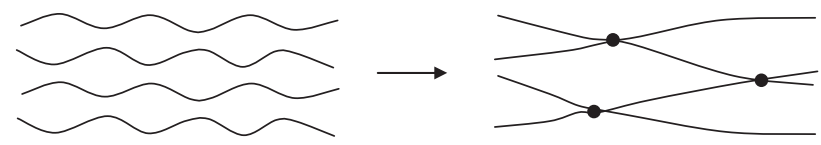

Figure 21.11 Schematization of the two types of changes in polymeric chain skeleton during chemical ageing. 
where $M_{n 0}, M_{W 0}, M_{n}$ and $M_{W}$ are respectively the number and weight average molar masses before and after ageing.

One can see that, if $S>4 X$, chain scission predominates. Alternatively, if $S<4 X$, crosslinking predominates.

As long as the material remains $100 \%$ soluble, molar mass measurements (by steric exclusion chromatography (CES), rheometry, etc.) can be carried out to evaluate such chemical changes. Saito's theory (Eqs (21.4) and (21.55)) allows the determination of $S$ and $X$.

However, if crosslinking predominates, the material reaches a gel point $\left(M_{W} \rightarrow \infty\right.$ and $X \rightarrow X_{g}$, whereas $M_{n}$ remains finite) and an insoluble fraction appears. Beyond the gel point, the determination of soluble fraction $\left(w_{s}\right)$ or elastic properties in rubbery state are the most common analytical ways of characterization. Then, Charlesby's theory can be used to determine $S$ and $X$ :

$$
w_{S}+w_{S}^{1 / 2}=\frac{S}{2 X}-\frac{1}{M_{W 0} X}
$$

This relationship is easily applicable if $S$ and $X$ are linear functions of time, as is generally the case in radiochemical ageing at relatively high dose rates.

Consequences of a Random Chain Scission In linear polymers, in the absence of crosslinking, Eqs (21.4) and (21.5) lead to:

$$
\begin{aligned}
P I & =2+\frac{M_{W}}{M_{W 0}}\left(P I_{0}-2\right) \\
\text { i.e.: } P I & =2+\frac{P I_{0}-2}{1+S M_{W 0} / 2}
\end{aligned}
$$

where $P I_{0}$ and $P I$ are the polydispersity indexes respectively before and after ageing.

Since $M_{W}$ decreases during degradation, $I P$ tends towards 2 at long ageing time (large conversion ratio), see Figure 21.12. Thus, if $P I$ is initially close to 2 , it will remain constant during degradation by random chain scission: $P I_{0} \approx P I \approx 2$. Such a simplification is current in the field of polymer chemical ageing.

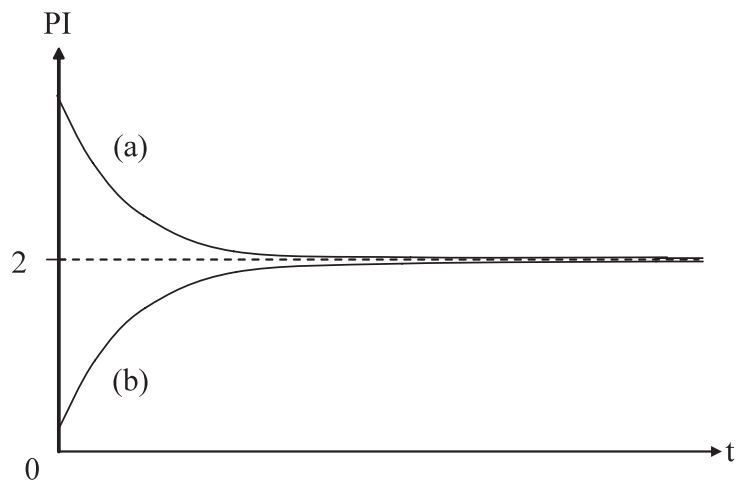

Figure 21.12 Shape of the variation of polydispersity index (PI) as a function of ageing time for a linear polymer undergoing a random chain scission: (a) $\mathrm{PI}_{0}>2$. (b) $\mathrm{PI}_{0}<2$. 


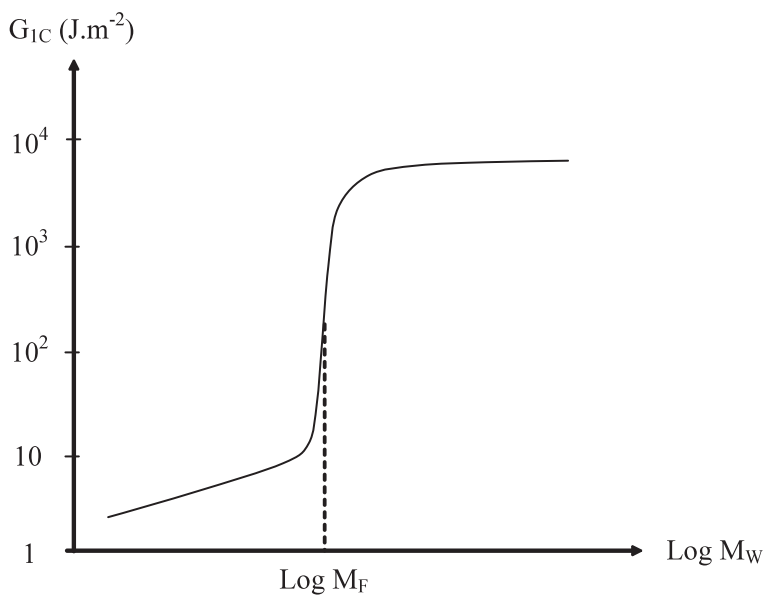

Figure 21.13 Shape of the variation of toughness $\left(G_{1 C}\right)$ for a linear polymer as a function of its weight average molar mass $\left(M_{W}\right)$.

Fracture properties of virgin linear polymers, for instance their toughness $G_{1 C}$ or their ultimate elongation $\varepsilon_{R}$, increase abruptly of several decades when their molar mass $M_{W}$ becomes higher than a critical molar mass $M_{F}$ (cf. Figure 21.13), this latter being closely related to the rheological molar mass $M_{C}$ [15].

One can thus expect that, during degradation by random chain scission, the fracture properties of an initially ductile/tough linear polymer decrease catastrophically when its molar mass becomes lower than the $M_{F}$ value. Below $M_{F}$, the polymer behaves like a wax or an eggshell, depending on its stiffness. It can no longer be used in mechanical applications owing to its extremely high brittleness.

Now, let's consider a random chain scission process occurring in a linear polymer with a constant rate $r_{S}$. The number of chain scission is just:

$$
S=r_{S} t
$$

Assuming that $P I_{0} \approx P I \approx 2$, Eq. (21.4) can be rewritten as:

$$
M_{W}=\frac{2 M_{W 0}}{2+r_{S} M_{W 0} t}
$$

The variations in time of the molar mass and ultimate elongation are schematized in Figure 21.14. These results call for the following comments:

1 Whereas $S$ is a linear function of time, $M_{W}$ is an hyperbolic function of time;

$2 M_{W}$ is a continuously decreasing function of time, whereas $\varepsilon_{R}$ falls abruptly when $M_{W}=M_{F}$.

As a general rule, embrittlement occurs prematurely. As an example, let's consider the two application cases that are hydrolysis at ambient temperature of PA11 pieces in offshore applications and radio-oxidation at ambient temperature of PE used as electrical insulation in cables for nuclear power plants. For such polymers, typical molar masses $\left(M_{W 0}\right.$ and $\left.M_{F}\right)$ are reported in Table 21.1. From these values, the critical number of chain scissions per mass unit, responsible for the polymer embrittlement $\left(\varepsilon_{R} / \varepsilon_{R 0}<50 \%\right)$ has been calculated 


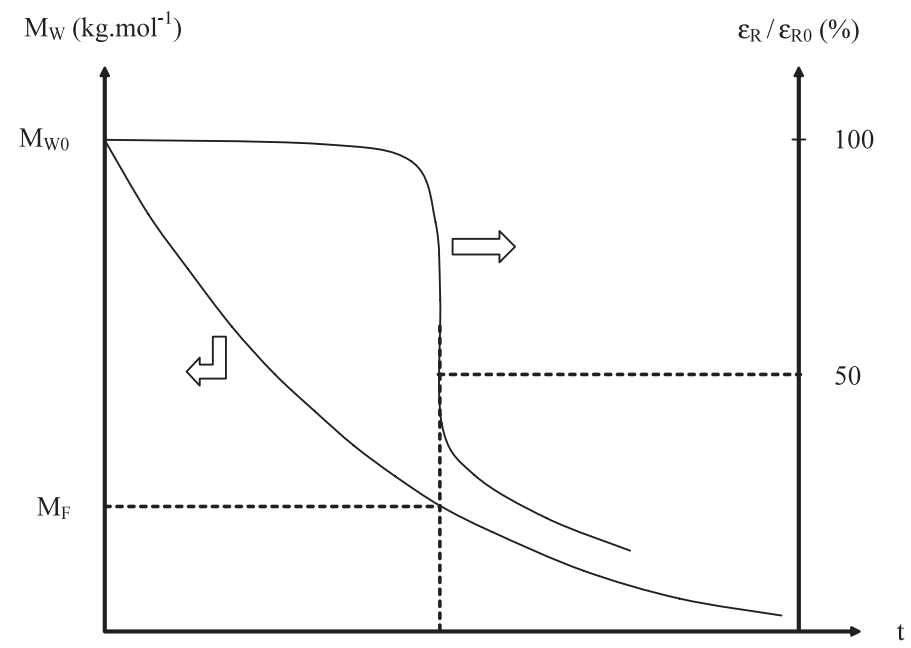

Figure 21.14 Shape of the variation in ageing time of weight average molar mass $\left(M_{W}\right)$ and ultimate elongation $\left(\varepsilon_{R}\right)$ for a linear polymer subjected to a random chain scission process.

from:

$$
S_{F}=\frac{2}{M_{F}}-\frac{2}{M_{W 0}}
$$

It is compared to the concentration of reactive sites susceptible to undergo a chain scission process (i.e. hydrolyzable amide groups for PA11 and oxidizable methylene groups for PE) in each polymer:

$$
C_{m}=\frac{1}{m}
$$

where $m$ is the molar mass of the monomer units: $m=0.183 \mathrm{~kg} \cdot \mathrm{mol}^{-1}$ for PA 11 , and $0.014 \mathrm{~kg} \cdot \mathrm{mol}^{-1}$ for PE.

The resulting conversion ratio $Y_{S}$ (at the molecular scale) of the chain scission process has been also reported in Table 21.1.

$$
Y_{S}=\frac{C_{m}}{S_{F}}
$$

Table 21.1 Embrittlement criteria for some common polymers. $M_{W 0}$ and $M_{F}$ are respectively initial and critical weight average molar masses; $S_{F}$ is the critical number of chain scission; $C_{m}$ is the concentration of potentially reactive sites and $Y_{S}$ is the resulting conversion ratio (at the molecular scale) of the chain scission process.

\begin{tabular}{llccccc}
\hline Polymer & Chemical ageing & $M_{W 0}\left(\mathrm{~kg} \cdot \mathrm{mol}^{-1}\right)$ & $M_{F}\left(\mathrm{~kg} \cdot \mathrm{mol}^{-1}\right)$ & $S_{F}\left(\mathrm{~mol}^{\mathrm{kg}} \mathrm{kg}^{-1}\right)$ & $C_{m}\left(\mathrm{~mol}_{\mathrm{kg}} \mathrm{kg}^{-1}\right)$ & $Y_{S}(\%)$ \\
\hline PA11 & Hydrolysis & 50 & 30 & $2.710^{-2}$ & 5.5 & 0.5 \\
PE & Oxidation & 150 & 70 & $1.510^{-2}$ & 71.4 & 0.02 \\
\hline
\end{tabular}


One can see that embrittlement occurs for a very low number of chain scissions and thus, a very low conversion ratio of reaction usually inaccessible by conventional spectroscopic techniques (infrared spectroscopy, nuclear magnetic resonance spectroscopy, etc.). This means that molar mass characterization (by steric exclusion chromatography or rheometry) is much more sensitive than such techniques for characterizing chain scission in the hypothesis that it is the only process at play.

Elastic and yield properties are almost not altered at such low conversion ratio. From then on, one can understand that chemical ageing-induced embrittlement constitutes a real domain of interest for many researchers. Whatever the importance of other use properties, no application can tolerate that the geometrical integrity of pieces be altered because of cracking.

Photo-oxidation affects only a thin superficial layer directly exposed to solar radiation, for instance a few dozens of micrometers in the case of epoxies [16]. In such chemical ageing types, local embrittlement can generate a network of microcracks without altering the global fracture behaviour of pieces. However, micro-cracking alters the surface aspect of pieces and may therefore have a significant economical impact for some applications, for instance in the case of varnishes of automotive bodies [17], for which aesthetic questions are particularly important.

In polymer networks (vulcanized elastomers, thermosets), random chain scission leads to a destruction of elastically active chains (EAC). Typically, at low conversion ratios of reaction, for a quasi-ideal network with a functionality $f \geq 4$ :

$$
v=v_{0}-S
$$

where $v_{0}$ and $v$ are the concentrations in EAC respectively before and after ageing.

These quantities can be determined from measurements of elastic properties in rubbery state:

$$
v=\frac{E}{3 \rho R T}=\frac{G}{\rho R T}
$$

where $E$ and $G$ are respectively the Young's and shear moduli (expressed in Pa), $\rho$ is the volumic mass (in $\mathrm{kg} \cdot \mathrm{m}^{-3}$ ), $R$ the perfect gas constant and $T$ the absolute temperature (in $K$ ).

$$
S=\frac{1}{3 \rho R T}\left(E_{0}-E\right)=\frac{1}{\rho R T}\left(G_{0}-G\right)
$$

Chain scission leads to a decrease of elastomers modulus. On the contrary, they have no direct consequences on glassy thermosets modulus. Fracture properties tend to decrease catastrophically, but structure-property relationships are not yet well known in this domain.

Consequences of Crosslinking In linear polymers, in the absence of chain scission, $M_{W}$ and $M_{n}$ increase, $P I$ increases, and $P I$ and $M_{W} \rightarrow \infty$ when the material reaches a gel point $\left(X \rightarrow X_{g}\right)$ :

$$
X_{g}=\frac{1}{2 M_{W 0}}
$$


One can see here also that gelation corresponds to a very low conversion ratio of reaction. Since it is necessary that two reactive sites encounter to weld two macromolecules, the conversion ratio at gel point is:

$$
Y_{g}=\frac{1}{M_{W 0}}
$$

As an example, in the case of a PE having an initial molar mass $M_{W 0}=150 \mathrm{~kg} \cdot \mathrm{mol}^{-1}$, one obtains: $Y_{g} \approx$ $6.610^{-3}$ mol. $\mathrm{kg}^{-1}$, whereas the concentration in potentially reactive sites (methylene groups) is $10^{4}$ times higher. Of course, some properties, in particular the flow properties in melt state, vary far before the gel point. In particular, the viscosity in melt state increases rapidly and the Newtonian plateau tends to disappear.

The ultimate elongation decreases progressively, whereas the stress at break increases, at least in the early period of exposure, for elastomers. In the case of thermosets, glass transition temperature and thus, yield stress increase, making yielding less and less competitive with brittle fracture.

\subsubsection{Diffusion Phenomena in Chemical Ageing}

Oxidation and hydrolysis, both being very common chemical ageing types, involve the penetration of a small reactive molecule (oxygen, water) initially present in the surrounding environment, into the material. One can thus define two characteristic times: one for diffusion, other for chemical reaction, for elaborating a simplified approach of the problem.

In the case of diffusion, the characteristic time can be defined such as:

$$
t_{D}=\frac{L^{2}}{D}
$$

where $L$ is the sample thickness and $D$ is the coefficient of reactive molecule diffusion.

In the case of reaction, one can use the following definition:

$$
t_{R}=\frac{C}{r_{C}}
$$

where $C$ is the equilibrium value of reactant concentration into the polymer, and $r_{C}$ is the rate of reactant consumption by the chemical reaction in the superficial layer of the material where its concentration is actually C.

From the equality of the critical times for diffusion and reaction, one can deduce a critical distance $L_{C}$ in the material as:

$$
\text { Then: } L_{C}=\left(\frac{D C}{r_{C}}\right)^{1 / 2}
$$

If the sample thickness is $L \ll L_{C}$, diffusion is rapid enough against reaction and, at any time $t$, the whole sample thickness is saturated in reactant. $r_{C}$ is depth independent. On the contrary, if the sample thickness is $L \gg L_{C}$, all the reactant is consumed in a superficial layer of thickness $L_{C}$ and the material core remains intact. The comparison of the global degradation rates of samples of different thicknesses allows the construction of the graph presented in Figure 21.15, which allows the determination of the value of $L_{C}$.

One can distinguish two distinct kinetic regimes of reaction, depending on the material thickness, as depicted in Figure 21.15: 


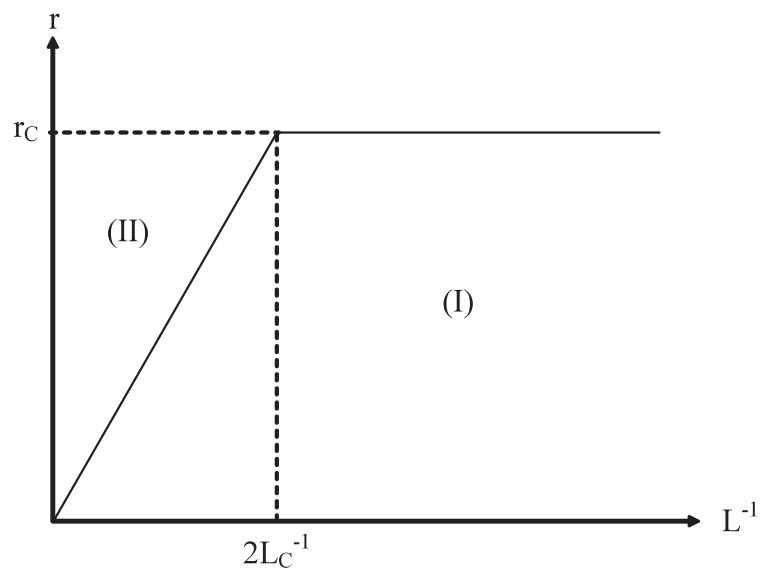

Figure 21.15 Shape of the variation, against reciprocal sample thickness $\left(L^{-1}\right)$, of global degradation rate $(r)$ in the hypothesis that both faces of the sample are exposed to diffusing species.

1 Regime (I), corresponding to thin samples, is not controlled by diffusion. The global degradation rate is thickness independent.

2 Regime (II) is diffusion controlled. The global degradation rate is proportional to the reciprocal thickness since the thickness of the degraded layer $L_{C}$ is thickness independent.

The value of the boundary between the two regimes is $2 L_{C}$ if the penetration of the reactive molecules takes place through both sample faces, as is usually the case.

For a more rigorous and more detailed approach to the problem, it is necessary to derive the relationship $r_{C}=r(C)$, expressing the rate of reactant consumption as a function of its concentration in relatively thin samples $\left(L \ll L_{C}\right)$, from a kinetic analysis of the corresponding mechanistic scheme. The rate of variation of the local reactant concentration in a relatively thick sample $\left(L \gg 2 L_{C}\right)$ can be determined from the following balance equation:

$$
\frac{\partial C}{\partial t}=D \frac{\partial^{2} C}{\partial z^{2}}-r(C)
$$

where $z$ is the spatial coordinate for an infinite plate (e.g. the depth beneath the sample surface).

The numerical resolution of this equation gives access to the distribution, in the sample thickness, of reactant concentration and its variation against time: $C=f(z, t)$.

In the case of hydrolysis, $r(C)$ takes, in general, the mathematical form of a pseudo-first-order reaction:

$$
r(C)=K C
$$

On the contrary, in the case of oxidation processes, $r(C)$ can be expressed, in a first approximation, by an hyperbolic function:

$$
r(C)=\frac{a C}{1+b C}
$$


where $a$ and $b$ are parameters to be expressed as functions of the rate constants of elementary reactions constituting the radical chain oxidation reaction.

As a general rule, chemical reactions are temperature dependent. In the scale equation (Eq. (21.21)) issued from a simplified approach of the problem, the equilibrium concentration of reactant $C$ is generally temperature independent, whereas the diffusion coefficient $D$ obeys an Arrhenius law:

$$
D=D_{0} \exp \left(-\frac{E_{D}}{R T}\right)
$$

where $D_{0}$ and $E_{D}$ are respectively the pre-exponential factor and the activation energy for reactant diffusion.

It has been shown that the temperature variation of the rate of reactant consumption in the superficial layer of the material, $r_{C}$, can be satisfyingly approximated by an Arrhenius law:

$$
r_{C}=r_{C 0} \exp \left(-\frac{E_{r}}{R T}\right)
$$

where $r_{C 0}$ and $E_{r}$ are respectively the pre-exponential factor and the activation energy for reactant consumption.

Finally, one can see that $L_{C}$ obeys also an Arrhenius law:

$$
\begin{aligned}
L_{C} & =L_{C 0} \exp \left(-\frac{E_{L}}{R T}\right) \\
\text { with: } L_{C 0} & =\left(\frac{D_{0} C}{r_{C 0}}\right)^{1 / 2} \text { and } E_{L}=\frac{1}{2}\left(E_{D}-E_{r}\right)
\end{aligned}
$$

In most of the cases of thermal oxidation and hydrolysis, $E_{D}<E_{r}$ so that $E_{L}$ is negative. Thus, $L_{C}$ is a decreasing function of temperature.

In the case of irradiation-induced chemical ageing, for instance radio and photo-oxidations, $C$ and $D$ are light intensity independent and Eq. (21.24) remains valid. At the opposite, it can be demonstrated that the variation against light intensity $I$ of $r_{C}$ can be satisfyingly approximated by a simple power law:

$$
r_{C} \propto I^{\alpha}
$$

with $\alpha=1 / 2$ in the simplest models.

Thus, the thickness of the oxidized layer is a slowly decreasing function of dose rate given by:

$$
L_{C} \propto I^{-\alpha / 2}
$$

The fact that the thickness of degraded layer is, in general, a decreasing function of the 'severity' of ageing conditions has been systematically observed by many authors.

\subsubsection{Consequences of Superficial Degradation on Use Properties}

Degradation gradients, resulting from a diffusion control of chemical reaction kinetics, play an important role from a mechanical point of view. Schematically, an initially ductile / tough and homogeneous polymer sample is progressively transformed into a binary structure, the sample core remaining ductile/tough, whereas the superficial layer becomes brittle and thus, highly sensitive to cracking. A superficial crack can cross rapidly 


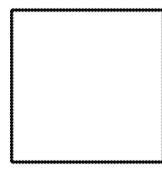

(0)

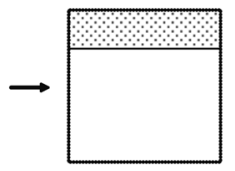

(I)

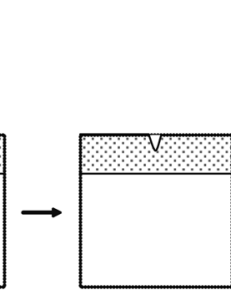

(II)

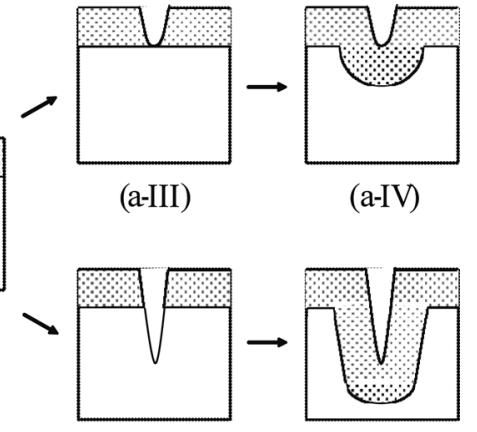

(b-III)

(b-IV)

Figure 21.16 Schematization of chemical ageing-induced cracking. Sample zones where the polymer chemical structure has been changed are represented in grey. (0) Initial (virgin) sample; (I) Superficial degraded layer below its embrittlement threshold; (II) Superficial degraded layer close to its embrittlement threshold; (a-III) Crack having reached the skin-core interface and having blunted; (b-III) Crack having crossed the interface; $(a-I V)$ and (b-IV) Propagation of oxidation front towards deeper layers.

the whole superficial layer thickness and reach the skin-core interface. At this stage, two scenarii can take place (Figure 21.16):

(a) crack tip blunts and remains restricted to the sample superficial layers

(b) crack crosses the interface and propagates in the core. Since cracks constitute a preferred path for small reactive molecules penetration, the degradation front moves towards deeper layers. A secondary degraded layer, of the same thickness as the primary one, forms beyond the crack tip, etc. Following this scheme, failure will ultimately occur, even without any external loading.

In a first approach, one can consider that the brittle superficial layer is equivalent to a 'natural' notch with the same depth. Fracture mechanics [18] predicts that there is a critical notch depth below which the notch does not initiate the material failure. This depth depends on the material toughness, this latter depending, in turn, on the rate of crack propagation. As an example, in the case of PE oxidation, this depth is of the order of magnitude of $100 \mu \mathrm{m}$ [19].

It is thus expected that rapid chemical ageing, leading to very small thicknesses of degraded layer, will have less effect on the material fracture behavior than slower ones. This general trend has been also observed by many authors.

\subsubsection{Mechanistic Schemes}

Chemical ageing is a complex phenomenon, but decomposable into a more or less high number of elementary reactions obeying relatively simple kinetic laws: Arrhenius law, proportionality to light intensity, etc.

Although chemical ageing proceeds through molecular scale (monomer unit) processes, we have shown in the previous sections that use properties of materials depend also on structural events occurring at the upper scales, in particular at the macromolecular (macromolecular skeleton) and the macroscopic ones (skin-core structure).

The objective of the present section is to give a rough description of the main chemical reactions occurring in a polymer during exposure to an aggressive chemical environment. 
Table 21.2 Orders of magnitude of the dissociation energy $\left(E_{D}\right)$ of main polymer chemical bonds.

\begin{tabular}{ll}
\hline Chemical bond & $E_{D}\left(\mathrm{~kJ} \cdot \mathrm{mol}^{-1}\right)$ \\
\hline aromatic C-C & 510 \\
C-F & 470 \\
aromatic C-H & 465 \\
aliphatic C-H & $325-425$ \\
aliphatic C-C & $300-380$ \\
C-O & 340 \\
C-Cl & 320 \\
C-Si & 300 \\
C-N & 290 \\
C-S & 275 \\
S-S & 260 \\
O-O & 150 \\
\hline
\end{tabular}

\subsubsection{Intrinsic Chemical Stability}

In the absence of reactive molecules (water, oxygen) in the environment of exposure, polymers can decompose because of the own material instability, this latter being governed by thermochemical factors. It involves, in general, radical reactions initiated by the breakdown of the weakest chemical bonds (i.e. characterized by the lowest dissociation energy) of macromolecules.

Aromatic $\mathrm{C}-\mathrm{C}$ and $\mathrm{C}-\mathrm{H}$ bonds and $\mathrm{C}-\mathrm{F}$ bonds (dissociative energy $E_{D}>450 \mathrm{~kJ} . \mathrm{mol}^{-1}$ ) are the most stable (Table 21.2). Polymers only composed of such chemical bonds are called, on purpose, 'thermostable polymers'. Polyimides and fluorinated polymers, e.g. polytetrafuoroethylene (PTFE), are examples of thermostable materials.

Aliphatic $\mathrm{C}-\mathrm{C}$ bonds are all the more unstable than the carbon atoms are more substituted, see for instance two series of bonds ranked by decreasing dissociation energy:

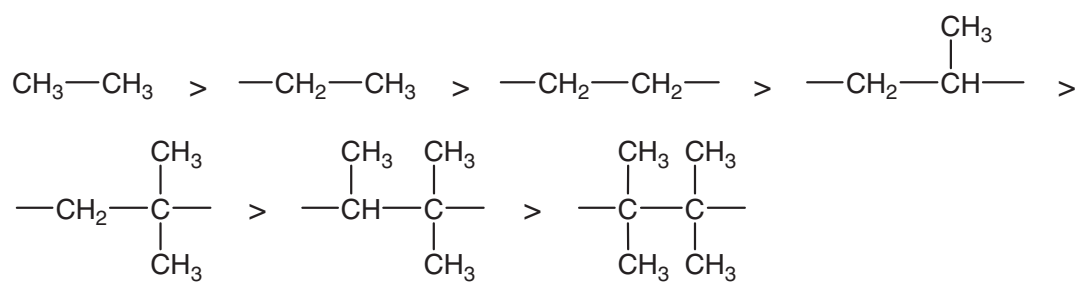

Thus, polymers containing tetrasubstituted carbons, such as polyisobutylene (PIB), poly(methyl methacrylate) (PMMA), poly $\left(\alpha\right.$ methyl styrene) $(\mathrm{P} \alpha \mathrm{MS})$, etc, are particularly unstable $\left(E_{D}=300-320 \mathrm{~kJ} \cdot \mathrm{mol}^{-1}\right)$. Also, polymers containing heteroatoms, for instance poly(oxy methylene) (POM), poly(ethylene terephthalate) (PET), poly(vinyl chloride) (PVC), sulphur vulcanized polyisoprene (SVPIP), etc, are also particularly unstable (Table 21.2).

However, the global thermal stability does not depend only on the presence of weak chemical bonds. It depends also on the ability of radicals to initiate a chain propagation reaction. As an example, in the case of polyethylene (PE), radicals deactivate easily by disproportionation:

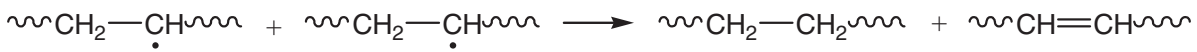


In the case of polymers containing tetrasubstituted carbons, as well as POM, radicals initiate a sequential (zip) elimination of monomer units:

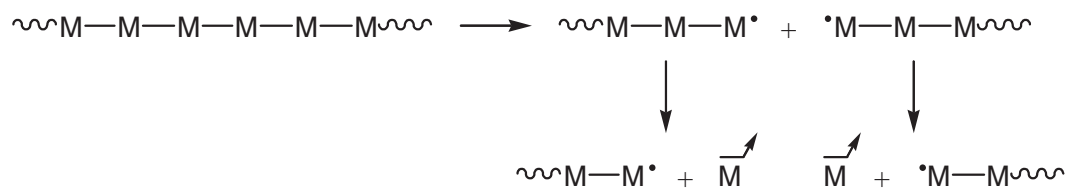

This reaction is called a 'depolymerization'. This is actually a weak point as polymers which depolymerize have the lowest thermal stability.

PVC undergoes a sequential (zip) elimination of its lateral groups:

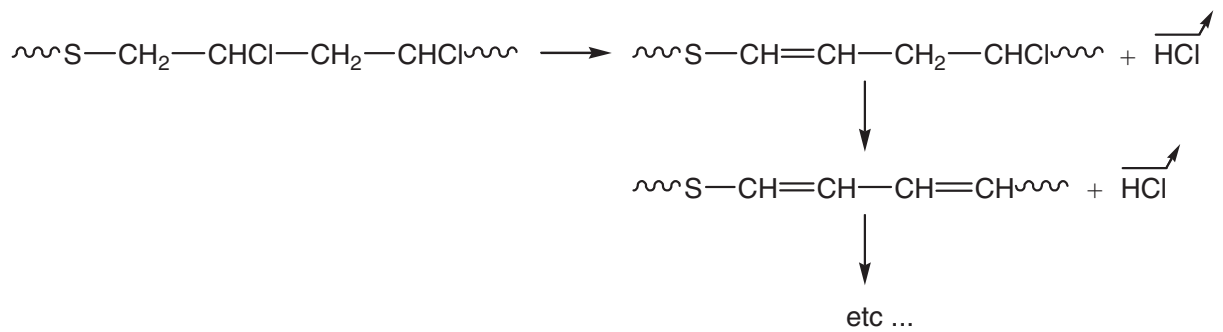

where $\mathrm{S}$ is a structural irregularity (for instance, a tertiary or an allylic chloride formed during polymer synthesis) making the first elimination step easier.

In the case of step-by-step processes, such as PE thermal degradation, there is no well-known way of stabilization by acting on the macromolecular structure. On the contrary, in the case of chain reactions (depolymerization, sequential elimination of volatile products like, for instance, $\mathrm{HCl}$, etc.), stabilization can be envisaged through several routes:

1 Suppressing the 'weak points' by chemical modification during polymer synthesis (blocking of terminal groups in the case of $\mathrm{POM}$ ) or by stabilization reaction during the processing operation (using stabilizers for PVC).

2 By interrupting efficiency the propagation by using an adequate co-monomer, for instance in the case of POM:

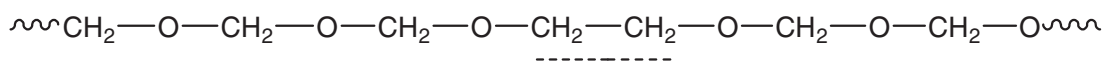

The insertion of ethylene units (as underlined in the above scheme) in the macromolecule by copolymerization allows slowing down depolymerization. POM copolymers can be easily distinguished from POM homopolymer: the former presents a melting point at about $165^{\circ} \mathrm{C}$, i.e. about $10-20^{\circ} \mathrm{C}$ lower than the latter.

\subsubsection{Oxidative Ageing}

General Aspects In most of the cases of thermal, radiochemical or photochemical ageing, the main ageing cause is the polymer oxidation by atmospheric oxygen. In all cases, degradation propagates through a radical chain reaction, the only difference being the initiation step. Thus, polymer oxidation kinetics can be 
described satisfactorily by a general mechanistic scheme called 'standard scheme', established in the 1940s by the RAPRA co-workers [20]:
(1) Polymer $\rightarrow \mu \mathrm{P}^{\circ}$
$\left(\mathrm{r}_{1}\right)$
(2) $\mathrm{P}^{\circ}+\mathrm{O}_{2} \rightarrow \mathrm{PO}_{2}^{\circ}$
$\left(k_{2}\right)$
(3) $\mathrm{PO}_{2}{ }^{\circ}+$ Polymer $\rightarrow$ Per $+\mathrm{P}^{\circ}$
$\left(k_{3}\right)$
(4) $\mathrm{P}^{\circ}+\mathrm{P}^{\circ} \rightarrow$ inactive products
$\left(k_{4}\right)$
(5) $\mathrm{P}^{\circ}+\mathrm{PO}_{2}^{\circ} \rightarrow$ inactive products
$\left(k_{5}\right)$
(6) $\mathrm{PO}_{2}{ }^{\circ}+\mathrm{PO}_{2}{ }^{\circ} \rightarrow$ inactive products $+\mathrm{O}_{2}$
$\left(k_{6}\right)$

In the 'standard scheme' of oxidative ageing, (1) corresponds to the initiation step, (2) and (3) to propagation steps; (4)-(6) to termination steps. $\mathrm{P}^{\circ}$ and $\mathrm{PO}_{2}{ }^{\circ}$ refer respectively to alkyl and peroxy radicals; Per refers to peroxide groups; $\mu$ is the formation yield of radicals in the initiation step; $r_{1}$ is initiation rate and $k_{i}$ are rate constants.

The initiation step (1) can be thermal, photochemical or radiochemical. It can result from a reaction involving directly lateral groups of monomer units (radiochemical ageing), or unstable pre-existing products like hydroperoxides and peroxides Per (particularly in thermal and photochemical ageing).

The propagation reaction is composed of two elementary steps (2) and (3):

(2) Oxygen addition to alkyl radicals: It is a very fast process and thus, practically structure- and temperatureindependent. The corresponding rate constant is very high: $k_{2}=10^{8}-10^{9} 1 . \mathrm{mol}^{-1} \cdot \mathrm{s}^{-1}$ [21].

(3) Peroxyl radicals reaction with the polymer: this is a largely much slower process, which is structuredependent. In saturated hydrocarbon polymers, for instance polyethylene (PE) and polypropylene (PP), it corresponds to hydrogen atom abstraction. In this case, Per is a hydroperoxide group (POOH). The corresponding rate constant is very low: $k_{3}=10^{-3}-10^{-1} 1 . \mathrm{mol}^{-1} \cdot \mathrm{s}^{-1}$ at ambient temperature (cf. Table 21.3). In polyenic elastomers, for instance polybutadiene (PBD) and polyisoprene (PIP), step (3) can be an addition to double bonds. Thus, Per can be a peroxide group POOP. The corresponding rate constant is also very low: for instance $k_{3}=10^{-1}-10 \mathrm{~s}^{-1}$ at ambient temperature in the case of the intramolecular addition (cf. Table 21.3).

Table 21.3 Propagation reactions of oxidation in some common hydrocarbon polymers and corresponding rate constant $\left(k_{3}\right)$ value at ambient temperature. PP: polypropylene; PE: polyethylene; PDB: polybutadiene; PIP: polyisoprene.

\begin{tabular}{llll}
\hline Propagation & Polymer & $k_{3}\left(\mathrm{l} . \mathrm{mol}^{-1} \cdot \mathrm{s}^{-1} \mathrm{or} \mathrm{s}^{-1}\right)$ & Source \\
\hline Hydrogen atom & PP & $1.0 \times 10^{-3}$ & {$[22]$} \\
abstraction & PE & $2.4 \times 10^{-3}$ & {$[22]$} \\
& PBD & $4.9 \times 10^{-3}$ & {$[23]$} \\
$\begin{array}{l}\text { Intramolecular } \\
\begin{array}{l}\text { addition to } \\
\text { double bonds }\end{array}\end{array}$ & PIP & $5.2 \times 10^{-2}$ & {$[1]$} \\
$\begin{array}{l}\text { Intermolecular } \\
\text { addition to }\end{array}$ & PBD & $6.1 \times 10^{-1}$ & {$[1]$} \\
double bonds & PBD & 2.7 & {$[23]$} \\
\hline
\end{tabular}


In the absence of antioxidants, the terminations of radicals are bimolecular processes. At relatively low temperature, like ambient temperature, termination rate constants of reactions given in the above scheme classify in the following order [24]:

$$
k_{4}>k_{5} \gg k_{6}
$$

(corresponding activation energies classify in the reverse order), mainly because $\mathrm{P}^{\circ}$ radicals can propagate by hydrogen abstraction: $\mathrm{P}^{\circ}+\mathrm{PH} \rightarrow \mathrm{PH}+\mathrm{P}^{\circ}$. This transfer reaction does not influence the whole oxidation kinetics (except for polyenic elastomers), but provides a simple explanation to the relatively high mobility of alkyl radicals and thus, the high $k_{4}$ value.

However, at moderate to high temperature, reactions (5) and (6) lead mainly to unstable peroxides (POOP). As a result, Eq. (21.29) is no longer valid. When $T>200^{\circ} \mathrm{C}$, for instance, it is rather observed [25, 26]:

$$
k_{4}>k_{5} \approx k_{6}
$$

Over the past half century, the most delicate and debated subject has been initiation. Initiation processes can be very varied and complex. They depend on the polymer nature (PH) and the way energy is brought to the system (by temperature or radiation). Though intermediate steps are not always totally known (often due to unsuccessful identification of chemical evolution during this step), the common output of this (these) $\mathrm{step}(\mathrm{s})$ is the formation of alkyl radicals $\mathrm{P}^{\circ}$.

For sake of simplicity, we consider the case of oxygen excess (relatively thin polymer sample exposed to a relatively high oxygen pressure). In this case, all the $\mathrm{P}^{\circ}$ radicals are almost instantaneously transformed into $\mathrm{PO}_{2}{ }^{\circ}$ and then their probability to participate to reactions other than reaction (6), in particular reactions (4) and (5), is negligible. As a result, the 'standard scheme' reduces to four reactions (1, 2, 3 and 6). One can distinguish two important initiation processes, as outlined below.

Case 1: Initiation at a Constant Rate In the case of radiochemical ageing (high energy provided), the main source of radicals is polymer radiolysis, i.e. the breakdown of lateral bonds of monomer units. In the case of PE, radiolysis leads to the formation of very reactive radicals $\mathrm{H}^{\circ}$ which recombine rapidly by hydrogen atoms abstraction:

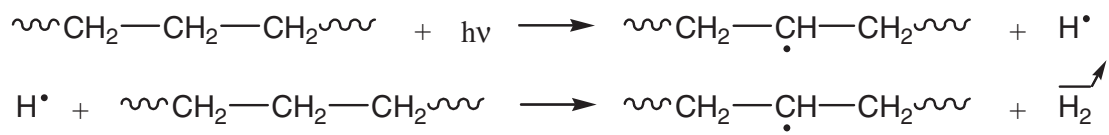

Thus, the corresponding balance initiation can be written:

$$
\text { (1) } \mathrm{PH} \rightarrow \mathrm{P}^{\circ}+\frac{1}{2} \mathrm{H}_{2} \quad\left(r_{1}\right)
$$

where the initiation rate $r_{1}$ is proportional to the dose rate $I$ according to:

$$
r_{1} \approx 10^{-7} G_{1} I
$$

and $G_{1}$ is the radical yield expressed in number of radicals $\mathrm{P}^{\circ}$ per $100 \mathrm{eV}$ absorbed, of the order of magnitude of $1-10$ for saturated hydrocarbon polymers [27]. 


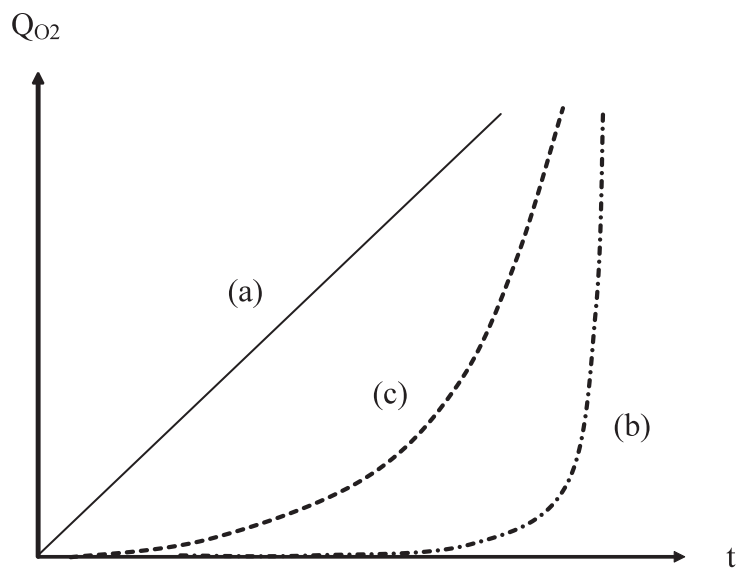

Figure 21.17 Shape of oxidation kinetic curves in the case of initiation (a) at constant rate (e.g. radiochemical ageing); (b) by bimolecular Per decomposition (thermal ageing); and (c) by unimolecular Per decomposition (case of many photochemical ageings).

In this case, the reaction reaches rapidly a steady state and oxidation products accumulate with a constant rate (cf. Figure 21.17). One can easily demonstrate that initiation and termination products form with a rate proportional to $r_{1}$. As an example, if ketones are formed with a yield $\gamma_{K}$ in termination:

$$
r_{K}=\gamma_{K} k_{6}\left[\mathrm{PO}_{2}^{\circ}\right]^{2}=\frac{\gamma_{K}}{2} r_{1}
$$

On the contrary, propagation products form with a rate proportional to the square root of $r_{1}$, for instance:

$$
r_{P e r}=k_{3}\left[\mathrm{PO}_{2}^{\circ}\right][\mathrm{PH}]=k_{3}[\mathrm{PH}]\left(\frac{r_{1}}{2 k_{6}}\right)^{1 / 2}
$$

Finally, oxygen is absorbed with a rate:

$$
r_{O 2}=k_{2}\left[O_{2}\right]\left[P^{\circ}\right]-k_{6}\left[\mathrm{PO}_{2}^{\circ}\right]^{2}=\frac{r_{1}}{2}+k_{3}[\mathrm{PH}]\left(\frac{r_{1}}{2 k_{6}}\right)^{1 / 2}
$$

Case 2: Initiation by Decomposition of Peroxides In the case of thermal and photochemical ageing (lower energy provided), the problem is significantly more complex. Chemical bonds of common industrial polymers have rarely a dissociation energy lower than $260 \mathrm{~kJ}^{-\mathrm{mol}^{-1}}$ (Table 17.2) and thus decompose only at high temperatures (typically, at $T>250^{\circ} \mathrm{C}$ ) or at high irradiation intensities (for instance, under $\gamma$ irradiation in nuclear environments). However, in the previous sections it has been seen that oxidation leads to the formation of two main propagation products: hydroperoxides $\mathrm{POOH}$ and peroxides POOP, noted $\mathrm{Per}$, of which the activation energy of $\mathrm{O}-\mathrm{O}$ bond is very low: $E_{D} \approx 150 \mathrm{~kJ} \cdot \mathrm{mol}^{-1}$. Such chemical groups are thermally and photochemically unstable, in particular in use conditions. 
It cannot be totally excluded that an 'extrinsic specie' generates also primary radicals (e.g. decomposition of structural irregularities or direct oxygen-polymer reaction). But it can be easily demonstrated that its contribution to initiation is very limited: the corresponding initiation rate is initially very low and vanishes rapidly (as soon as the 'extrinsic specie' concentration vanishes). As a result, in all cases, initiation by $\mathrm{Per}$ decomposition rapidly becomes the main source of radicals. From a kinetic modeling point of view, the following approach is usually adopted: initially present 'extrinsic specie' is replaced by a kinetically equivalent initial $\mathrm{Per}$ concentration: $[\mathrm{Per}]_{0}$. In this case, initiation involves two reactions:

$$
\text { (1) } \quad \delta \text { Per } \rightarrow \alpha \mathrm{P}^{\circ}+\beta \mathrm{PO}_{2}^{\circ} \quad\left(k_{1}\right)
$$

with: $\alpha=2$ and $\beta=0$ for unimolecular decomposition $(\delta=1)$

$\alpha=1$ and $\beta=1$ for bimolecular decomposition $(\delta=2)$

Then, the initiation rate depends on $\mathrm{Per}$ concentration:

$$
r_{1}=k_{1}[\mathrm{Per}]^{\delta}
$$

Such an oxidation mechanism is called a 'close-loop mechanism', because it produces its own initiator product $(\mathrm{Per})$. The resulting kinetic curves present an induction period followed by a sharp auto-acceleration, preceding a steady state. The auto-acceleration step is much more progressive in its initial phase when $\mathrm{Per}$ decomposition is unimolecular (the case of many photochemical ageings) (cf. Figure 21.17).

According to analytical models, the duration of the induction period is given by:

$$
\begin{aligned}
t_{i} & \approx \frac{5}{2 k_{1}} \quad(\text { unimolecular decomposition }) \\
t_{i} & \approx \frac{1-\ln Y_{0}}{K} \quad \text { (bimolecular decomposition) }
\end{aligned}
$$

where $K=k_{3}[\mathrm{PH}]\left(\frac{k_{1}}{k_{6}}\right)^{1 / 2}$ and $Y_{0}=\frac{[\mathrm{Per}]_{0}}{[\mathrm{Per}]_{S}}$

Moreover, in steady state, the Per concentration is:

$$
[\mathrm{Per}]=-\delta k_{1}[\mathrm{Per}]^{\delta}+k_{3}\left[\mathrm{PO}_{2}^{\circ}\right][\mathrm{PH}]=\left[\frac{k_{3}^{2}[\mathrm{PH}]^{2}}{\delta^{2} k_{1} k_{6}}\right]^{1 / \delta}
$$

and the rate of oxygen consumption is:

$$
r_{\mathrm{O} 2}=k_{2}\left[O_{2}\right]\left[\mathrm{P}^{\circ}\right]-k_{6}\left[\mathrm{PO}_{2}^{\circ}\right]^{2}=\frac{2}{\delta^{2}} \frac{k_{3}^{2}[\mathrm{PH}]^{2}}{k_{6}}
$$

The kinetic analysis of oxidation mechanisms shows that the kinetic behavior depends, in both cases 1 and 2, on two main factors:

1 An extrinsic factor, that is to say an external factor to polymer structure: initiation rate $r_{1}$ or initiation rate constant $k_{1}$;

2 An intrinsic factor characterizing the polymer oxidisability: ratio $\frac{k_{3}[\mathrm{PH}]}{\sqrt{k_{6}}}$. 
Table 21.4 Orders of magnitude of the dissociation energy $\left(E_{D}\right)$ of $\mathrm{CH}$ bonds.

\begin{tabular}{|l|l|}
\hline C-H bond & $\mathbf{E}_{\mathbf{D}}\left(\mathbf{k J} . \mathrm{mol}^{-1}\right)$ \\
\hline- & 465 \\
\hline$-\mathrm{CH}_{3}$ & 414 \\
\hline$-\mathrm{CH}_{2}-\mathrm{CH}_{2}-$ & 393 \\
\hline$-\mathrm{CH}-$ & 378 \\
\hline$-\mathrm{O}-\mathrm{CH}_{2}-$ or $-\mathrm{N}-\mathrm{CH}_{2}-$ & 376 \\
\hline$\triangle \mathrm{C}=\mathrm{CH}-\mathrm{CH}_{2}-$ & 335 \\
\hline
\end{tabular}

According to some authors [22], there is a linear relationship between $\log k_{3}$ and the dissociation energy $E_{D}$ of $\mathrm{CH}$ bonds. Thus, in a first approach, polymer stability to oxidation can be roughly estimated from the reactivity of the $\mathrm{CH}$ bonds involved (cf. Table 21.4).

The following global trends can be deduced:

1 Polymers without $\mathrm{CH}$ bonds such as poly(tetra fluoro ethylene) (PTFE), or containing exclusively aromatic $\mathrm{CH}$ bonds such as poly(ether ether ketone) (PEEK), poly(ether sulfone) (PES) and polyimides (PI), are stable to oxidation.

2 Polymers containing exclusively methyl $\mathrm{CH}$ bonds such as poly(dimethyl siloxane) (PdMS), or containing methyl and methylene $\mathrm{CH}$ bonds such as: poly(methyl methacrylate) (PMMA), polycarbonate (PC) and polyethylene (PE), are moderately stable.

3 Polymers containing methyne $\mathrm{CH}$ bonds such as polypropylene (PP), or methylene $\mathrm{CH}$ bonds in a $\alpha$ position of an heteroatom such as: poly(methylene oxide) (POM), polyamides (PA) and amine crosslinked epoxy (ACE), are relatively unstable.

4 Finally, polymers containing allylic $\mathrm{CH}$ bonds such as polyisoprene (PIP) and polybutadiene (PBD), are highly unstable.

The above section has treated ageing kinetics only through intrinsic stability of polymeric chains. There are actually other factors of determining importance when dealing with polymers in real conditions, with two examples given:

Oxygen diffusivity play an important role through provision of one of the necessary reactants: It is about three orders of magnitude higher in elastomers than in glassy polymers. Thus, at equal reactivity, glassy polymers will appear much more stable because their oxidized layer will be much thinner.

At least another factor plays an important role for use properties: the polymer sensitivity to chemical events resulting from oxidation. As an example, mechanical embrittlement occurs in polypropylene (PP) for a number of chain scissions ten times higher than in an amorphous polymer. Thus, at equal reactivity, according to this mechanical endlife criterion, PP will be ten times less stable than an amorphous polymer.

Resulting Macromolecular Changes If oxygen is in excess (no termination involving alkyl radicals), in general, chain scission is largely predominant over crosslinking. The $\beta$ scission of alkoxy radicals $\mathrm{PO}^{\circ}$ is probably the most frequent source of chain scissions in polymer oxidation. $\mathrm{PO}^{\circ}$ radicals come from the decomposition of $\mathrm{Per}$ groups:

$$
\delta P e r \rightarrow \alpha \mathrm{PO}^{\circ}+\beta \mathrm{PO}_{2}^{\circ}
$$


or from the nonterminating bimolecular combinations of peroxy radicals $\mathrm{PO}_{2}{ }^{\circ}$ :

$$
\begin{array}{ll}
\mathrm{PO}_{2}^{\circ}+\mathrm{PO}_{2}^{\circ} & \rightarrow \mathrm{PO}-\mathrm{O}-\mathrm{O}-\mathrm{OP} \rightarrow\left[\mathrm{PO}^{\circ} \mathrm{OP}\right]_{\text {cage }}+\mathrm{O}_{2} \\
{\left[\mathrm{PO}^{\circ} \mathrm{OP}\right]_{\text {cage }}} & \rightarrow \text { inactive products } \\
{\left[\mathrm{PO}^{\circ} \mathrm{OP}\right]_{\text {cage }}} & \rightarrow 2 \mathrm{PO}^{\circ}
\end{array}
$$

$\beta$ scission can be schematized as follows:

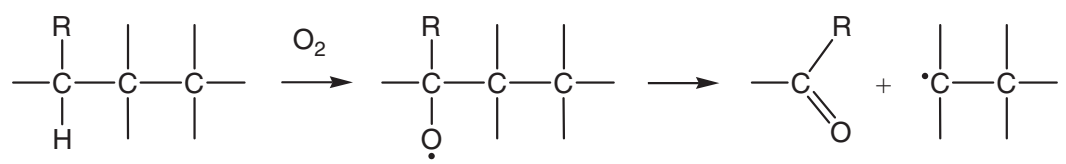

It leads to an alkyl radical and a carbonyl group. If $\mathrm{R}=\mathrm{H}$ (case of polyethylene (PE)), this latter is an aldehyde, whereas if $\mathrm{R}=\mathrm{CH}_{3}$ (case of polypropylene (PP)), it is a ketone. Note that this reaction is in competition with hydrogen abstraction:

$$
\mathrm{PO}^{\circ}+\mathrm{PH} \rightarrow \mathrm{P}-\mathrm{OH}+\mathrm{P}^{\circ}
$$

Thus, if the chain scission process occurs with a yield $\gamma_{S}$ in initiation or non terminating combination, the corresponding chain scission rate is:

$$
\begin{array}{ll}
r_{S}=\gamma_{S 6} k_{6}\left[\mathrm{PO}_{2}^{\circ}\right]^{2}=\frac{\gamma_{S 6}}{2} r_{1} \quad \text { (radiochemical ageing) } \\
r_{S}=\gamma_{S} k_{1}[\mathrm{Per}]^{\delta}=\frac{\gamma_{S}}{\delta^{2}} \frac{k_{3}^{2}[\mathrm{PH}]^{2}}{k_{6}} \quad \text { (thermal and photochemical ageing) }
\end{array}
$$

Crosslinking predominates only in some polyene elastomers, for instance in polybutadiene (PBD), in which the addition of peroxy radicals to double bonds is, partly, intermolecular:

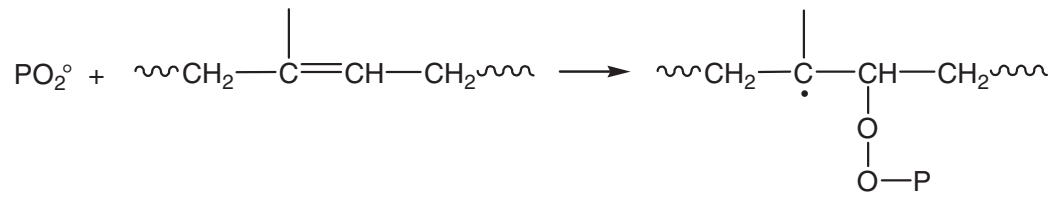

Stabilization Chain reactions, such as radical chain oxidation, especially if they produce their own initiator product, present a serious drawback. It can be called the 'butterfly effect': small causes lead to great consequences. However, this drawback is transformed into advantage if one envisages a stabilization by additives, in particular antioxidants. Since oxidation starts very slowly, from a low concentration of reactive species (Per, radicals), and then auto-accelerates, one can envisage to inhibit it with a low concentration of adequately chosen antioxidants. On the contrary, if an extrinsic initiation, with a constant rate, occurs, antioxidants lose their efficiency [28].

In the case of a 'closed-loop' oxidation mechanism, there are two possible ways of stabilization:

(a) A reduction of initiation rate by decomposing hydroperoxides by a nonradical way:

(7) $\mathrm{POOH}+\mathrm{Dec} \rightarrow$ inactive products $\left(k_{7}\right)$ 


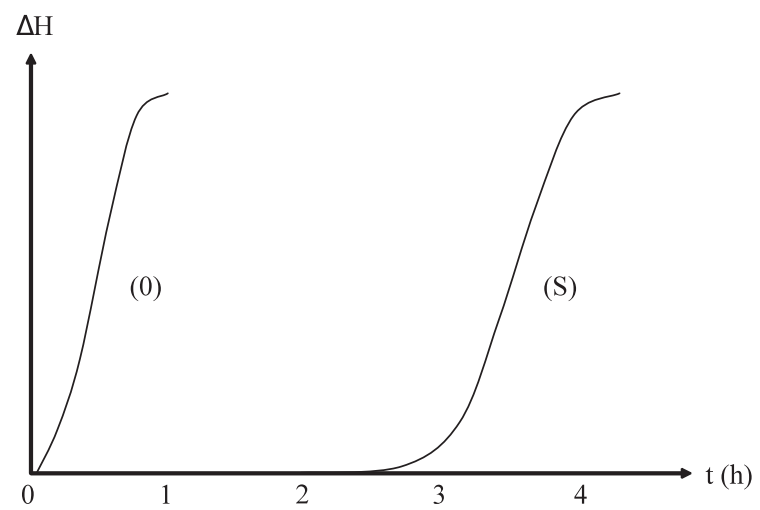

Figure 21.18 Shape of DSC thermograms in pure oxygen at $190^{\circ} \mathrm{C}$ for nonstabilized polyethylene (0), and the same polyethylene stabilized by $0.3 \%$ in weight of a phenol-phosphite mixture (S) [32]. In both cases, heating from ambient temperature to $190^{\circ} \mathrm{C}$ has been made under nitrogen and oxygen has been admitted in the cell after system thermal equilibrium.

In this case, $D e c$ is an organic sulphur or phosphite. Antioxidants belonging to this first family can be called 'preventive antioxidants'.

(b) A reduction of the propagation rate, i.e. an increase of the termination rate, by capturing radicals $\left(\mathrm{P}^{\circ}\right.$ and $\mathrm{PO}_{2}^{\circ}$ ):

(8) $\mathrm{P}^{\circ}+>\mathrm{NO}^{\circ} \rightarrow>\mathrm{NO}-\mathrm{P}\left(k_{8}\right)$

In this case, $\mathrm{R}-\mathrm{NO}^{\circ}$ is a nitroxy radical. This latter can be directly incorporated to the polymer during processing or formed by oxidation of an hindered amine (HAS) preliminary incorporated.

(9) $\mathrm{PO}_{2}^{\circ}+\mathrm{AH} \rightarrow \mathrm{POOH}+$ inactive products $\left(k_{9}\right)$

Here, AH is a hindered phenol or an aromatic amine. Such molecules give easily their hydrogen atom to peroxy radicals, because its dissociation energy is smaller than that of hydrogen atoms of saturated hydrocarbon polymers. As an example, in the case of hindered phenols, the dissociation energy of A- $\mathrm{H}$ bond is about $335-355 \mathrm{~kJ} \mathrm{~mol}^{-1}$ (see, for instance [29, 30, 31]).

Antioxidants belonging to this second family are called 'chain breaking antioxidants' or, more simply, 'terminators', for the sake of shortness.

In the case of polyolefins, one can obtain an efficient stabilization with antioxidant weight fractions lower than $0.5 \%$. In the case of polyene elastomers, antioxidants are, in general, used in concentrations higher than $1 \%$. The most commonly-used method to evaluate the antioxidant efficiency consists in determining the oxidation induction time (OIT) at high temperature in pure oxygen (Figure 21.18).

The efficiency of antioxidants depends also on several physical factors:

1 Their solubility into the polymer. If this latter is too low, the concentration threshold necessary to have an optimized stabilization cannot be reached. Numerous antioxidants are constituted of a long hydrocarbon chain, for instance thiodipropionates:

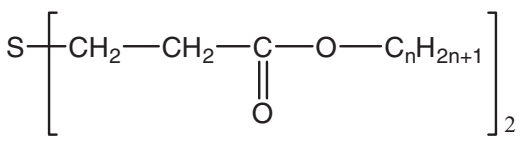

with $n=12$ (Dilauryl thiodipropionate (DLTDP)) or 18 (Distearyl thiodipropionate (DSTDP)). 
2 Their diffusivity into the polymer. If this latter is too high, their loss by migration can be too important and greatly reduce its efficiency. The solution consists in increasing their molar mass (hydrocarbon chain grafting, oligomer antioxidants, etc.) to reduce their diffusivity.

3 Their interactions with other additives. As an example, the efficiency of some antioxidants can decrease dramatically in the presence of fillers. Such antagonistic effects have been reported for associations of preventive antioxidants (hindered phenols or aromatic amines) and carbon black [33]. Some authors have attempted to provide an explanation. For some of them, the surface of fillers could catalyze the antioxidant oxidation [34]. For others, antioxidants could be neutralized by adsorption at the surface of fillers [35].

As another example, the efficiency of some antioxidants can increase strongly in the presence of another antioxidant. Such synergetic effects are well known for the past 50 years, for associations of preventive and terminator antioxidants, for instance for mixtures of sulphur + phenol, or phosphite + phenol. It is now currently used in industry (see, for instance [36]). For a long time, these synergetic effects have been interpreted in terms of chemical interactions between stabilizers or their by-products, for instance the regeneration of the phenol group by a reaction between the sulphur and phenol by-products [37], or more simply, by the fact that the sulphur reduces the hydroperoxide resulting from a stabilization step involving the phenol [39]. It can be easily demonstrated that, in fact, this synergetic effect results essentially from a simple kinetic effect due to the fact that each antioxidant acts in a different level of the radical chain process [28].

\subsubsection{Hydrolytic Ageing}

General Aspects Hydrolytic ageing results from chemical interaction between polymer and water. The main chemical event is a hydrolysis process which can be schematized as follows:

$$
\sim \mathrm{A}-\mathrm{B} \sim+\mathrm{H}_{2} \mathrm{O} \rightarrow \sim \mathrm{A}-\mathrm{OH}+\mathrm{H}-\mathrm{B} \sim
$$

In the most important practical cases, the broken chemical bond belongs to the macromolecular skeleton. Thus, each hydrolysis event is a chain scission. As a result, hydrolysis leads to polymer embrittlement at very low ratios of the reaction, like other chain scission processes studied previously, that justify the large amount of literature works dealing with chemical ageing of this type.

A water molecule participates to the hydrolysis of a polymer chemical group if it penetrates into the sample. In this case, it modifies also the polymer properties and leads to a physical ageing. There are cases of 'pure' physical ageing by water absorption in the case of hydrophilic (but no hydrolyzable) polymers. On the contrary, there is no 'pure' chemical ageing, because each chemical ageing is followed by plasticization or swelling phenomena that are relevant to physical ageing. This ageing type is reversible (as long as the material does not damage), a drying restores initial material properties. Chemical ageing, on the other hand, is irreversible (Figure 21.19).

Irreversible Hydrolysis Polycarbonate (PC), saturated linear polyesters (PET, PBT) and unsaturated polyesters (UP) crosslinked by styrene hydrolyze up to high conversion ratios, without observation of a significant slow down of reaction kinetics. For these polymers, one can consider, in a first approximation, that hydrolysis is irreversible. Thus:

$$
E+W \rightarrow A c+A l
$$




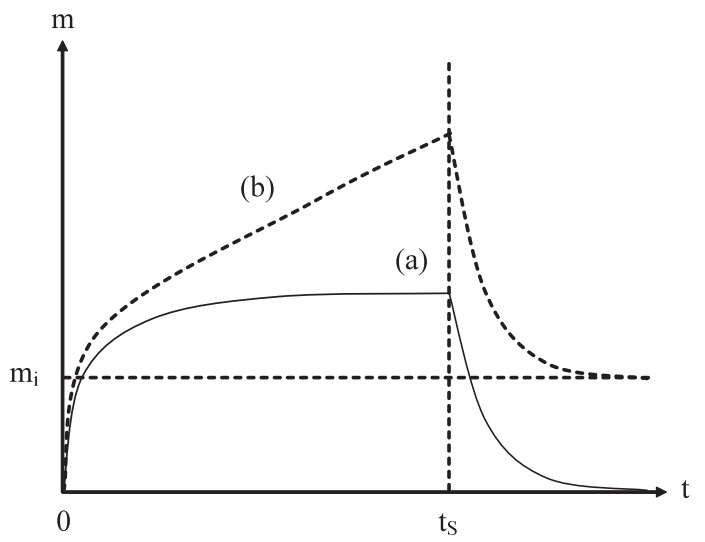

Figure 21.19 Shape of weight changes in humid $\left(t<t_{S}\right)$ and dried atmosphere $\left(t>t_{S}\right)$. (a) Case of 'pure' physical ageing: the weight gain tends towards an equilibrium value and, after drying, the material recovers its initial weight. Absorption and desorption rates are equal. (b) Case of hydrolysis (without extraction of small molecules from sample). Each hydrolysis event leads to a weight gain of $18 \mathrm{~g} \cdot \mathrm{mol}^{-1}$. An irreversible sample weight variation is induced after drying. Note that the sample weight can increase, decrease or vary nonmonotonically according to chemical ageing type.

where $E$ is an ester group, $W$ a water molecule, and $A c$ and $A l$ are respectively acid and alcohol groups resulting from hydrolysis.

Each hydrolysis event leads to a chain scission $(S)$. It can be thus written, in a nondiffusion controlled regime:

$$
\frac{d S}{d t}=k[E][W]=k\left([E]_{0}-S\right)[W]
$$

Since embrittlement occurs at a very low conversion ratio: $S \ll[E]_{0}$, hydrolysis does not modify much the polymer hydrophilicity:

$$
\frac{d S}{d t}=k[E]_{0}[W] \approx \mathrm{cst}
$$

Hence: $S=K t$

$$
\text { with } K=k[E]_{0}[W]
$$

Let's recall that the endlife criterion, corresponding to the ductile/brittle transition, is: $M_{W}=M_{F}$, and the corresponding critical number of chain scissions is:

$$
S_{F}=\frac{2}{M_{F}}-\frac{2}{M_{W 0}}
$$


Thus, the lifetime $t_{F}$ is:

$$
t_{F}=\frac{S_{F}}{K}=\left(\frac{2}{M_{F}}-\frac{2}{M_{W 0}}\right) \frac{1}{k[E]_{0}[W]}
$$

$k$ obeys an Arrhenius law. [W] is determined from the water chemical potential. In the case of a slightly hydrophilic material:

1 if the material is immerged in pure water:

$$
[W]=[W]_{S}
$$

where $[W]_{S}$ is the equilibrium value of water concentration into the material.

2 if the material is exposed to a humid atmosphere:

$$
[W]=[W]_{S} \frac{\mathrm{RH}}{100}
$$

where $\mathrm{RH}$ is the relative hygrometry.

Thus, in the general case:

$$
t_{F}=\left(\frac{2}{M_{F}}-\frac{2}{M_{W 0}}\right) \frac{1}{[E]_{0}} \frac{1}{k_{0} \exp -E / R T} \frac{100}{[W]_{S} \mathrm{RH}}
$$

with $\mathrm{RH}=100$ in the case of an immersion in pure water.

The above equation provides a nonempirical relationship between lifetime and three factors representing the effects of polymer structure, temperature and environment composition.

Reversible Hydrolysis In some cases, for instance PA11, hydrolysis is strongly autoretarded from the early period of exposure, and the sample weight tends towards an equilibrium value, that clearly indicates the existence of a reversible process:

$$
\begin{aligned}
A+W & \rightarrow A c+A m \quad\left(k_{H}\right) \\
& \leftarrow
\end{aligned}
$$

where, in this case, $A$ is the amide group, and $A c$ and $A m$ are respectively acid and amine groups resulting from hydrolysis.

One can write:

$$
\frac{d S}{d t}=k_{H}\left([A]_{0}-S\right)[W]-k_{R}\left([A c]_{0}+s\right)\left([A m]_{0}+s\right)
$$

It can be easily shown that the equilibrium molar mass $M_{n e}$ (when $t \rightarrow \infty$ ) is given by:

$$
M_{W e}=2\left(\frac{k_{R}}{k_{H}[A]_{0}[W]_{0}}\right)^{1 / 2}
$$



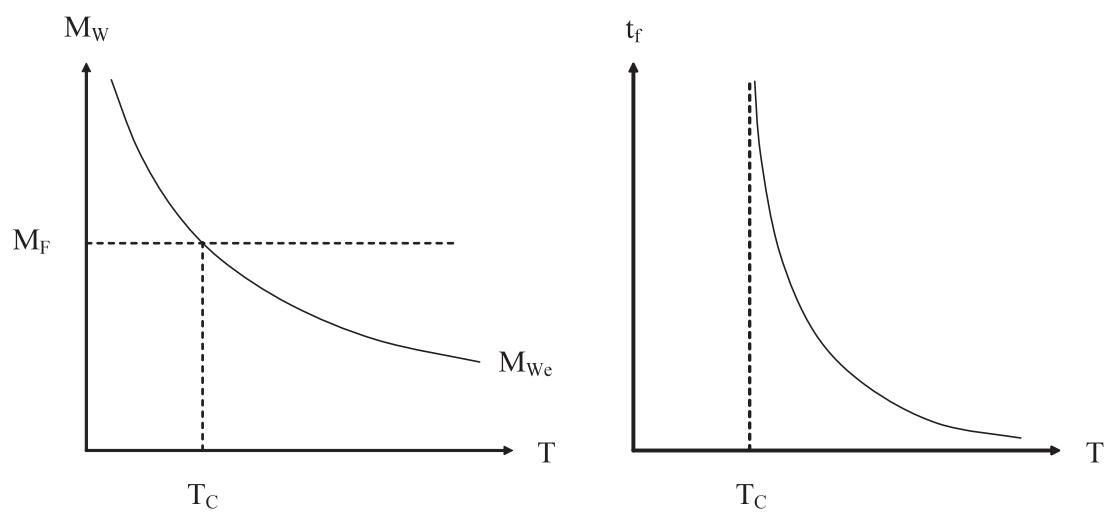

Figure 21.20 Molar mass-temperature map (left). Lifetime versus temperature (right).

The molar mass is a decreasing exponential function of temperature (Figure 19.20). One can thus distinguish two distinct cases:

1 If $T>T_{C}$, then $M_{W e}<M_{F}$. The material becomes brittle during its chemical ageing.

2 If $T<T_{C}$, then $M_{W e}>M_{F}$. The material never becomes brittle, but reaches an equilibrium. Lifetime is theoretically infinite.

In the case of $\mathrm{PA} 11, T_{C} \approx 80^{\circ} \mathrm{C}$.

\subsection{Impact of Multiphase Structure on Ageing Processes}

Two aspects of multiphase structures are considered in this section: on the one hand, the semicrystalline nature of polymers implies the existence of amorphous regions and regions of well-packed molecular chains, generally of higher density, with interfacial regions between them. The second important family is that of composites, in which charges of variable nature and shape are incorporated. Generally, such charges are inorganic materials and are not specifically directly involved in ageing. We consider here how the multiphase nature of materials may affect ageing processes described above.

\subsubsection{Structural Reorganization}

Structural recovery concerns the amorphous phase of polymeric materials. In semicrystalline polymers, molecular motions within the amorphous phase are hindered to some extent by the presence of the crystalline phase. As a consequence, the glass transition temperature usually increases with the crystalline degree, and its manifestation (by DSC, for example) is smeared out. Therefore, structural recovery is a second order problem in this kind of material.

The main changes in physical properties of semicrystalline polymers are therefore linked to variations of the crystalline degree itself. Such changes generally do not occur if the material is kept at a temperature below $T_{g}$. For higher temperatures, cold crystallization may occur, to an extent depending on the processing conditions of the material, in particular the cooling rate: a fast cooling rate freezes the material in a outof-equilibrium, disordered state. The molecular mobility prevents packing of macromolecules into crystal 


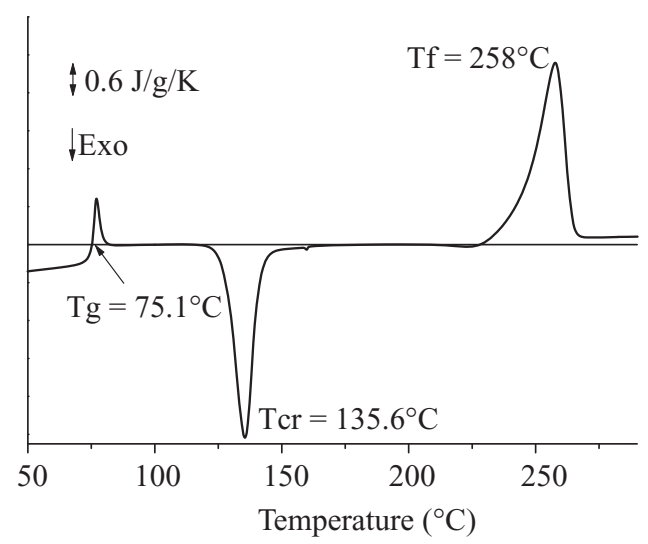

Figure 21.21 Example of cold crystallization process observed by DSC on PET. The material was initially quenched to room temperature providing so essentially an amorphous phase. On subsequent heating, the glass transition and associated recovery of structural relaxation (small endothermic peak) are observed, followed by cold crystallization at $135^{\circ} \mathrm{C}$ and melting at $258^{\circ} \mathrm{C}$.

structure. With time and/or in combination with a temperature increase, so-called cold crystallization occurs. This marginally concerns materials like polyethylene which crystallize very fast and can virtually not be obtained in an amorphous state. Materials like Poly(ethylene terephthalate) or Poly(ethylene naphthalate) can easily be obtained either in the amorphous or crystalline structures and are therefore prone to cold crystallization (cf. Figure 21.21).

Negative impact (that can be viewed as positive effects depending on the targeted application) of a crystalline degree increase can be as follows:

- increase in brittleness of the material

- loss of elasticity, increase in Young's modulus

- volume reduction due a crystalline phase more dense than the amorphous one, leading e.g. to void formation in the bulk, shrinkage, and decohesion from charges in a composite or from adjacent pieces

- change in material aspect (from transparent to translucent due to diffusion of light by crystallites)

- apparition of mechanical and dielectric losses due to the so-called $\alpha$-relaxation process (see e.g. [39] for the outlines of such a process).

A peculiarity of shrinkage effects in oriented polymeric films is worth mentioning here. Polyvinylidene fluoride (PVDF) is known for its ferroelectric properties. Forty years after the discovery of piezoelectricity in PVDF, it remains one of the best piezoelectric polymers available so far. For inducing ferroelectric properties to PVDF, uniaxial or biaxial mechanical drawing of films, to a ratio of the order of 4 , has to be carried out, inducing change in the crystalline phase from a nonpolar $\alpha$-phase to a polar $\beta$-phase. A high electric field is then applied to provide a macroscopic polarization to the films. The point is that when the material is heated to temperature of ca. $70{ }^{\circ} \mathrm{C}$ and above, shrinkage of the films occurs, implying motion of the crystalline regions. This results in a lowering of the macroscopic polarization and henceforth an ageing of the pyroelectric and piezoelectric properties [40]. The copolymerization of VDF units with TrFE (trifluoroethylene) produces directly crystallization in the polar phase, so avoiding drawing of the films and improving the thermal stability of the electroactive properties of the materials. 


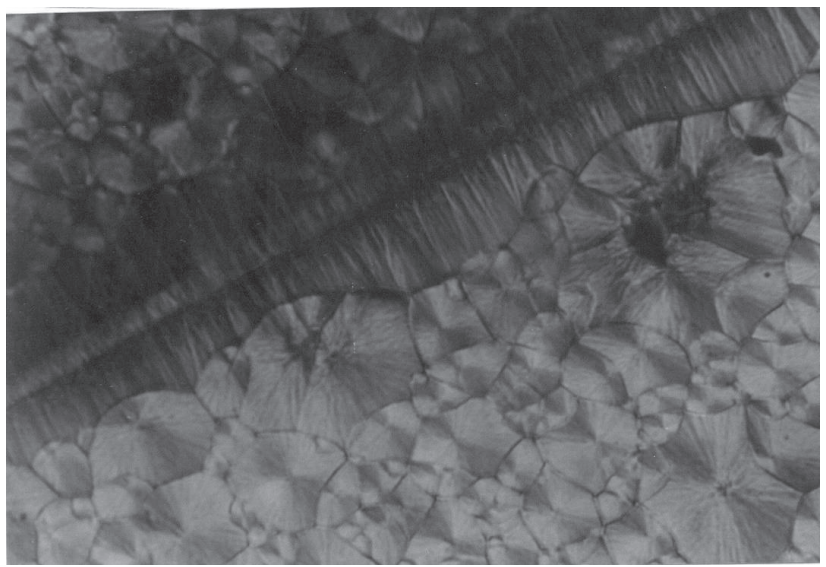

Figure 21.22 Example of a transcrystalline region formed around a fiber during crystallization of a PPS/carbon fiber composite.

In composite materials, structural recovery is concerned, to a first approximation, in the same way as for amorphous materials if micrometric scale particles are considered. In nanocomposites, i.e. composites containing nanometer scale particles, it can be anticipated that hindering of molecular motions occurs and impedes such effects, as it does for semicrystalline materials. Crystallization mechanisms can be significantly modified by the presence of charges. Indeed, two processes of crystallization germination are at play, which are homogeneous germination (self ordering of macromolecules acting as a core of the crystal or spherulite) and heterogeneous germination, i.e. crystal growth starting from a defect or from small crystals in surfusion. Therefore, charges themselves may act as 'defects' inducing crystal growth preferentially from the surface of such defects. Figure 21.22 shows an example of heterogeneous germination of Poly(phenylene sulphide) -PPS- on a Carbon fiber: very specific morphology, called transcrystalline region, is obtained in this case [41]. It results from a dense crystal nucleation on the fiber and a growth of crystalline lamella along radial directions of the fiber. It has been reported that the interface between the transcrystalline region and material bulk can constitute a weak point from the mechanical point of view in fiber-reinforced semicrystalline polymers.

\subsubsection{Diffusion Controlled Processes}

Generally speaking, diffusion processes tend to be slowed down when particles are incorporated into a matrix. Already geometrical considerations can support the statement. As diffusion proceeds through free volume occupancy, the presence of crystalline lamellae and/or of inorganic particles reduces the volume effectively available for diffusion since it concerns essentially amorphous phases. In addition, molecular motions are hindered by the presence of charges/crystallites and hence it can be anticipated that the diffusion coefficient for small molecules be lower in the amorphous phase of a semicrystalline material than in a purely amorphous material. There are, however, notable exceptions to this statement, particularly as regards water. In epoxyglass composites as an example, water tends to accumulate at the interfaces between matrix and filler and to form so-called water shells. If at this stage, chemical degradation is not discussed, the presence of water at such interfaces can have tremendous consequences, like a huge increase in dielectric losses, along with the formation of weak cohesive points. This is a critical issue for the mechanical resistance of the composite as it is expected that stresses are transferred between the two phases through this interface. Except for this 
specific case of water in epoxy-based composites which is dealt with specifically in the next section, it can be considered that composites are detrimental to diffusion, which can be used as a positive property.

\subsection{Practical Impact of Physical Ageing on Use Properties}

So far, we have dealt with ageing mechanisms in polymer and composites that appear under 'normal' ambient conditions, essentially as a combination of humidity, oxygen and temperature. In the following, we review issues associated with material evolution under functional stresses, i.e. specific environmental constraints that materials are supposed to sustain during the life of systems with which they are incorporated. In many instances, the impact of ageing mechanisms on mechanical properties has been discussed in the previous sections. Therefore, we shall consider only briefly these aspects therein. Given the extremely broad range of applications in which polymers are incorporated today, it is impossible to build an exhaustive review of those stresses. Instead, we propose to consider electrical insulating properties, an important application to our eyes in which the 'multiphase' nature of materials is exploited. The issues associated with the 'multiphase' nature of materials are discussed in relation to the demanding performances for such application.

\subsubsection{Water-Induced Mechanical Damages in Composites}

\subsubsection{Osmotic Cracking}

In the case of composites made of glass fibers and unsaturated polyester matrix (boat hulls, swimming pools, etc.), weight changes due to water absorption can present the peculiar shape illustrated in Figure 21.23.

This behavior can be explained as follows:

1 The initial stage corresponds to water absorption by the classical dissolution-diffusion mechanism. At time $t_{1}$, the material reaches its sorption equilibrium.

2 However, water reacts chemically with the polymer. Since this latter presents a lot of chain ends, chain scissions close to chain extremities lead to an accumulation of small organic molecules (monomers, oligomers). When these small molecules (which are more polar than the polymer) reach their solubility threshold into the polymer, they induce, at time $t_{2}$, a phase separation and generate micropockets of highly hydrophilic liquid. The water entrance in these micropockets generates an osmotic pressure and,

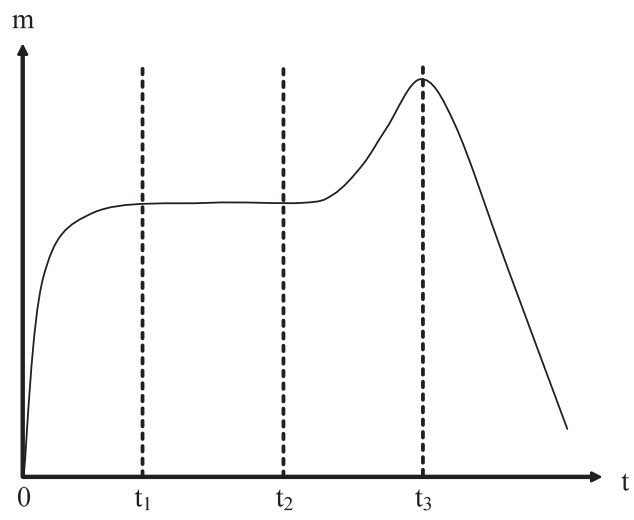

Figure 21.23 Shape of weight changes for a material composite subjected to an osmotic cracking. 
thus, a stress state which can initiate cracks at the micropocket surface. Between $t_{2}$ and $t_{3}$, the sample weight increases because cracks propagate and receive more and more water.

3 At time $t_{3}$, cracks coalesce and the solutes leave the sample, producing a quick drop in sample weight.

In the case of composite materials, cracks develop in sub-layers of the material and propagate parallel to the sample surface, which leads to blister formation. Material ability to generate blisters in the presence of water is essentially linked to the concentration of small molecules accumulating in the matrix. This concentration depends on three factors:

1 the concentration of initially present small molecules (in particular, the initiator of polymerisation and its reaction products)

2 the initial concentration of chain ends (all the more so high since the molar mass of initial linear unsaturated polyester is low)

3 the rate of polymer hydrolysis (leading to new chain ends).

The effect of these different factors has been checked experimentally (for instance, [42]).

\subsubsection{Interfacial Hydrolysis}

In some cases of composite materials based on matrices particularly resistant to hydrolysis (vinylester, nonhydrolyzable thermoplastics), humid ageing leads to an alteration of mechanical properties which cannot be attributed to a chemical interaction between water and matrix. Microscopic or mechanical analyses (for instance, resistance to interlaminar shearing) show the existence of a fiber-matrix debonding.

Moreover, the composite stability, in given conditions, depends closely on the nature of the coupling agent. Thus, hydrolysis reactions take place at the fiber-matrix interphase.

There are numerous experimental methods to support qualitatively this phenomenon. But, to our knowledge, there are no methods allowing quantifying, in an undisputable way, the numbers of chemical events responsible for this evolution. At the present time, there is no possibility to perform a fine kinetic analysis of this chemical ageing type.

\subsubsection{Ageing of Electrical Insulations}

Polymers are involved as electrical insulating materials in all the systems of electrical engineering, electrotechnics and electronics. Some examples of application and of materials used as insulations are given in Table 21.5. Criteria behind the selection of a given material depend on the intrinsic electrical performances expected (see below), on the resistance to other environmental stresses (mechanical, UV, temperature, humidity...), on the processability of the material and on economical factors. In the following, we detail the expected electrical properties and consider their evolution in bulk polymers and composites.

\subsubsection{Critical Electrical Properties}

Field Strength The most important electrical property for an electrical insulation is the field strength, i.e. the maximum electrical field the material can sustain without breakdown. Typical values are in the range 15 to $600 \mathrm{kV} / \mathrm{mm}$, depending on materials, on the stress form (DC, AC, impulse voltage) and on sample thickness. The property must be maintained during the service life of the material. The monitoring of field strength is often a way of evaluating, from the electrical standpoint, the resistance of material to degradation during 
Table 21.5 Field of application of some polymers and composites in Electrical Engineering.

\begin{tabular}{|c|c|c|c|}
\hline \multicolumn{2}{|c|}{ Electrical Engineering - Electrotechnics } & \multicolumn{2}{|c|}{ Electronics - Power electronics } \\
\hline Application & Materials & Application & Material \\
\hline High voltage cables & $\begin{array}{l}\text {-Polyethylenes, PVC } \\
\text {-Carbon black } \\
\text { reinforced polymers }\end{array}$ & Capacitors & $\begin{array}{l}\text { Polypropylene, PET, } \\
\text { polycarbonate, } \\
\text { polystyrene, } \\
\text { fluorinated polymers, } \\
\text { polysulfones }\end{array}$ \\
\hline Outdoor insulations & Epoxy, silicone & $\begin{array}{l}\text { Components } \\
\text { packaging }\end{array}$ & Silicone gels \\
\hline Power transformers & Silica-filled epoxies & $\begin{array}{l}\text { Power electronics } \\
\text { insulation }\end{array}$ & Polyimide \\
\hline Low voltage cables & $\begin{array}{l}\text { XLPE, EPR, PVC, ETFE } \\
\text { (fluorinated } \\
\text { copolymers), silicones }\end{array}$ & Circuit pressboards & $\begin{array}{l}\text { Fiberglass reinforced } \\
\text { epoxies }\end{array}$ \\
\hline Wires: varnishes & $\begin{array}{l}\text { Epoxydes, polyimides, } \\
\text { polyurethanes, } \\
\text { polyamides }\end{array}$ & & \\
\hline Bus bars & Epoxy/mica composites & & \\
\hline
\end{tabular}

accelerated ageing tests. Usually, the field strength is approached as a short-term process, i.e. ageing effects associated with the application of the stress are not considered. Three mechanisms of dielectric breakdown are worth mentioning here. These are: avalanche breakdown, electromechanical breakdown and thermal breakdown. Avalanche multiplication occurs at high field strengths when carriers acquire sufficient kinetic energy between collisions (scattering events) with the matrix to give a high probability of ionization, with the generation of more carrier. Thus a small current can be greatly multiplied by an increase in the number of carriers until it is sufficiently large to cause irreversible damage. This type of mechanism has been used to explain breakdown in oxidized films. Thermal breakdown occurs when the heat input cannot be balanced by the heat losses from the insulation either macroscopically, or more usually, in a small area. As power is dissipated by the insulation, heating occurs which usually causes an exponential increase in the electrical conductivity as more carriers become available for conduction. If the electrical stress is maintained, the current density increases in the area at elevated temperature. This serves to further increase the local temperature through Joule effect and hence the conductivity, and so thermal runaway may occur. Electromechanical breakdown occurs due to the electrostatic attraction of the electrodes which decreases the width of the insulation by an amount depending on the Young's modulus. If the applied voltage is maintained, the field increases due to the decrease in thickness, thereby increasing the attraction force (which varies as a square function of field) further.

Aside from the mechanism itself, the form of breakdown, either homogeneous or filamentary, is questioned. Contrarily to mechanical breakdown which requires extensive damage to definitively occur, electrical breakdown can be initiated and accomplished through only a weak channel through the insulation. In this respect, models have been developed for filamentary breakdown [4] of either a thermal or electromechanical nature. For the latest mechanism, the filamentary process appears much more likely than homogeneous ones. The mechanism has analogy with fracture mechanics in which a crack spontaneously propagates according to the Griffith's criterion. It is substantiated in some works by the correlation between field strength and 
Young's modulus for e.g. experiments at variable temperature [44]. Filamentary processes could be initiated from weak points in the bulk or at the surface of the material. Breakdown statistics tend to show that the probability of having a breakdown event increases with the volume of small. This tends to provide support to defect-induced initiation of breakdown as opposed to homogeneous processes.

The above processes are general and are not specific to polymers. Dielectric breakdown processes in polymers are complicated due to their highly complex structures. Therefore, much work has been devoted to clarifying the correlation between the breakdown characteristics and the properties inherent to polymers. Since condensed states of polymers change with temperature, the temperature dependence of their dielectric breakdown is of prime importance in analyzing their breakdown mechanisms. Roughly, it can be considered that breakdown at low temperature in polymers could be related to avalanche breakdown, basically because the two other processes become less probable. Thermal and electrical breakdown would be favored for high conductivity materials and soft materials, respectively.

Electrical Ageing We refer here to ageing processes in which the electrical stress is a necessary condition to ageing. All the cited mechanisms are expected to lead to premature breakdown in the long term. The more severe forms of degradation in this respect are water trees, partial discharges and electrical trees.

Water trees are of concern where the main application is submarine cables. It results from the application of an electrical stress combined with contact with an aqueous electrolyte. Water trees do not generally form interconnected channels. After the initiation stage, trees grow very rapidly at first and then the speed of growth progressively decreases. These features have been tentatively explained by a great number of mechanisms of electromechanical, electrochemical oxidation or electro-mechano-chemical nature, but in spite of this diversity none of the mechanisms are adequate to describe all the basic observations. The common scheme is that of a microvoid formation (initiation) followed by extension of that void along a privileged direction.

Electrical trees are generated in field-enhanced regions of the material that can be a void or a metallic conducting center (the typical experiment for observing it being a needle electrode). Electrical trees consist of connected channels (hollow tubules) a few $\mu \mathrm{m}$ in diameter, with branches tens of microns long. The walls of the channels can be carbonized and are only weakly conducting. They are not considered as intrinsic runaway breakdown mechanisms, rather a cumulative damage of the insulation leading to an enhancement of the failure probability.

Finally partial discharges are discharges occurring in the gas included in voids of some tens of $\mu \mathrm{m}$ size included in the polymer. Erosion of the internal surface of the voids by particles from the discharges leads to an enlargement of the void and eventually formation of trees.

In practice, the actual life of the insulation is actually not controlled by the extension of such voids, but by the time for partial discharges to be detected. Figure 21.24 provides a sketch for a possible ageing and breakdown scenario for a material free from preexisting voids. Considerable improvement in the reliability of high voltage cables has been obtained by improving the quality of products and, notably, in avoiding the presence of microvoids inside insulations. Actually, the fundamental question for electrical ageing of insulations is the nature of mechanisms leading to the formation of voids of size large enough to sustain a discharge process [45]. This criterion remains one of the most important quality control parameters in the production of such cables. Acceleration of electrons in regions of lower density in the material (free volume regions) followed by impact excitation on molecules is one way through which molecular-excited states can be produced and may lead to chemical degradation [46]. Along these lines, the actual reactivity of centers' localizing electrical charges (space charge) and the electrostatic and electromechanical energy stored around such centers are other lines of thought for explaining such phenomena.

Space Charges In the absence of voids, space charge-induced phenomena represent one of the most important lines of research regarding electrical ageing of insulation [47]. Space charges are electronic or 


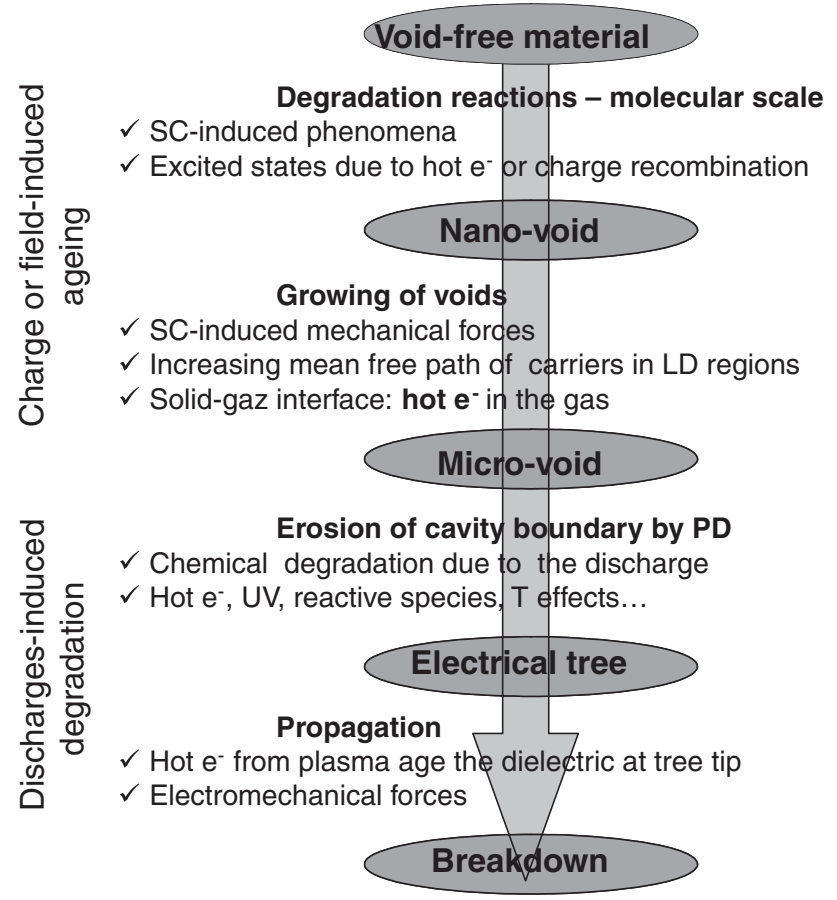

Figure 21.24 Sketch of a possible breakdown scenario in a void-free insulation material. Hot $\mathrm{e}^{-}$stands for hot electrons, i.e. electrons of kinetic energy of a few eV, able to excite molecules by impact. PD stands for partial discharges.

ionic carriers present in an insulation. Depending on their trapping energy, they can move under the effect of the electric field. Numerous techniques of detection of such charges have been developed during the last 30 years. The negative impact of space charges can be viewed from different standpoints:

- the acceleration of such charges may give rise to excitation of molecular centers as explained above

- recombination of charges of different polarity is another way of producing excited states in the insulation

- the accumulation of charges in some regions implies, in virtue of the Poisson's equation, changes in the actual electric field in respect of the design field. It implies necessarily regions of enhanced electric field that may induce premature breakdown of the insulation.

The last point is a critical issue for high voltage DC applications where, contrary to AC cases, there is no extensive experience regarding synthetic insulations, in particular for cables.

Surface Discharges Surface properties, for example, are essential features for outdoor insulations. Pollution of the surface leads to an increase of the conductivity, surface discharges processes (tracking discharges) and flashover. In a number of applications the surface has to be resistant to electrical discharges, in particular avoiding erosion of the material.

The environmental stresses are generally involved in failure associated with tracking discharges. That factor is often moisture that condenses on degraded or contaminated surfaces making them conduct current. Discharges occur when the current path is broken by drying. The discharge may cause local carbonization. 
More wetting causes further discharge, more carbonization, and finally, failure. The intensity of this effect of moisture is so dependent upon the surface condition that it is often separated from the aging mechanism and considered simply as a mode of failure [48].

Besides, moisture can contribute to a dominant mode of degradation that is very different from hydrolytic attack. When the environment includes rain, fog or any other form of condensate moisture, as well as voltage, a special type of deterioration occurs. It is the same process as in the failure mechanism, but now it determines the life and not simply the end of life, and the details are more important to understand. Leakage current flows across the surface and the heat generated causes a drying of the moisture film. A circumferential 'dry-band' forms, which partly interrupts the charge flow. Most of the applied voltage is supported by this dry-band, and arcs jump across it. These arcs are a type of discharge variously called 'arclets' or 'scintillation'. They are fed by the current through the moisture film, so that they are hotter than partial discharge would be. They impinge on the insulation surface, causing an ambient of fast moving, transient high temperature spots, each lasting a few ms. While they last, the atmosphere in the area of the arcs is an ionized plasma with a temperature of thousands of degrees. The surface of the insulation will react chemically with the constituents of the plasma, and degrade with the heat, causing a spot of carbon or other conductive residue to form on the material surface. This localized degradation may become an attachment point for the arc terminus (the cathode spot) so that the rate of degradation becomes self-accelerating. The degradation zone extends itself from one electrode to the other, and the former insulator ceases to be an insulator. This is one possible way to view 'surface tracking'. Essential features that must be maintained during the life of outdoor insulation are surface hydrophobicity in respect to water effects, and resistance to sunlight as a general requirement.

Other Issues A number of other electrical properties can be important for insulations and dielectrics, depending on the application. Among them, bulk dielectric properties, encompassing conductivity, permittivity and dielectric losses are fundamental parameters for such materials. Changes that can be observed here are induced more by environmental parameters such as humidity and water uptake, UV exposure, thermal stresses than by pure electrical ageing.

In the following, we give an overview of the impact of multiphase systems, being semicrystalline polymers and composites, on the properties described above.

\subsubsection{Ageing of Electrical Properties in Multiphase Systems}

Polymers in General Any ageing process able to produce voids within the insulation does have negative impact on the electrical performance. On the one hand, voids may initiate breakdown processes as explained above. On the other hand, diffusing species, among which water may segregate in such voids and produce therefore a composite material. Dielectric losses can be induced by such processes due to the difference in permittivity of the two phases. Such void-containing regions can be induced by mechanical stresses along with other chemical ageing processes producing crack. An incomplete cure of a resin can also form regions of low density. Post-curing of a resin produces volume contraction and is also able to produce voided materials; the same may happen with thermal cycling due to differences in thermal expansion coefficients.

Voltage stabilizers, i.e. additives for improving water and electrical treeing resistances and the electrical strength in polyethylene cables have been known for several decades but have not been widely used. The principles behind the stabilization mechanism are outlined as follows [49]. Stabilizers are generally aromatic groups being more easily ionized than the polymer chain, forming a stabile radical cation when an excited electron is captured. This means that rather than dissipating its energy by breaking bonds in the polymer chain the excited electron reacts with the stabilizer. This cation radical of the stabilizer will in turn be able to abstract 
energy from another excited electron or a radical center on the polymer, leaving them unexcited. When these steps have taken place the voltage stabilizer has been regenerated to its original state and no depletion of the stabilizer due to consumption will occur. The stabilization effect has been correlated to the ionization potential of such groups. However, the low solubility of the aromatic voltage stabilizers in polyolefins has been a dilemma. One of the main problems with voltage stabilizers until quite recently has been the depletion of stabilizer due to migration from the material, and hence a decrease in time of its efficiency. Alternative routes, such as grafting stabilizers on the chains [50] or design of molecules with higher stabilization efficiency have been proposed [51].

Thermal degradation generally goes with an increase in the conductivity of the material and is therefore detrimental to the insulation. The same also holds for UV ageing, particularly for surface insulation. Cracking, hardening and stresses cycling may induce decohesion between metallic parts and the insulation. From there, partial discharge can induce triggering and so erosion of the material.

Semicrystalline Polymers In some reports on the impact of microstructure on breakdown phenomena, it has been shown that spherulites may represent weak points of the insulation: discharge channels appearing along spherulites boundary [52]. This not completely surprising as the interfaces between spherulites are regions of lower density, that may also contain a high concentration of defects. It can be suspected that the current density is higher in these regions, so producing damage.

Crystalline regions constitute in general regions of lower mobility for charges. This, along with the fact that molecular motion is hindered so that free volume is reduced, leads to a lower conductivity for a semicrystalline polymer. The same holds for permittivity: the latter exhibits a significant step across the dielectric relaxation associated with the glass transition. An increase in crystallinity lowers the dielectric permittivity which can be detrimental for application as capacitors (energy storage). The counterpart is a decrease in the losses associated with the $\beta$-relaxation. An important consequence of the semicrystalline nature of polymers is the appearance of dielectric relaxation processes associated with paracrystalline regions ( $\alpha$-relaxation), at temperatures above $T_{g}$.

Composites There are numerous examples where, aside from economic benefits, composites bring a positive impact on insulation performance as a whole. Essentially, the gain obtained concerns thermal and mechanical properties, including a decrease in the thermal expansion coefficient and an improvement of the thermal conductivity and resistance to surface discharges. The latter effect can be understood by the strong resistance of inorganic particles (e.g. mica in transformer bars) to discharges, forming a protective layer and avoiding erosion. In outdoors insulations, silicone elastomers are used more and more with silica or ATH (alumina trihydrate) particles as fillers, one of the advantages of silicones being the possibility for low molecular weight polymeric chains to diffuse up to the surface of the insulation, so ensuring a regeneration of the surface properties (hydrophobicity recovery, incorporation of pollutants into the insulation bulk). See [53] for a review of ageing and recovery of silicone-based composites in the field of outdoor insulations.

There are, however, negative effects of the incorporation of fillers into composites. As with silicones, coatings develop leakage currents all the earlier that the filler content is high. Also, the incorporation of particles tends to decrease the hydrophobic properties. In silica-filled epoxy materials, the dielectric strength is divided by a factor of two in respect to the pure resin for $50 \%$ load. One of the reasons is that the particles are incompletely dispersed in the resin and therefore provide weak points. Also, water uptake can be important (of the order of $2 \%$ ), implying a tremendous increase in dielectric losses. The effect is much more pronounced in composites than in pure epoxy and is further increased in nanocomposites due to the large specific surface of particles $[54,55]$. Simple calculations indicate that the water is not dissolved in the resin but aggregates around silica particles forming all sorts of interfacial layers, and possibly decohesion. 
The last example concerns printed circuit boards (PCB), usually made of glass fiber-reinforced epoxies. Important issues are currently coming in relation to the increase in working temperature and voltage induced notably by the development of power electronics and by the trend towards high density integration of electronic circuits implying higher electric field applied to the insulation layer of a PCB. Aside from the surface effects that have been addressed for a long time, like oxidation of metallic layers and the formation of whiskers, new features have been revealed such as ionic migration in the bulk of the PCB [56] and possibly also migration of metal atoms and electrochemical processes along glass fibers from nearby contacts. Such effects may lead to short-circuit and failure of the device.

The above examples of ageing in polymer and composites used for their electrical insulating properties are in no way exhaustive and have been introduced for illustration purposes. Given the wide spread of application fields, of environmental conditions, of working stresses, of geometry and of nature of materials, weak points in terms of properties can be extremely variable. Concerning the relationships between the intrinsic electrical properties and the composite nature of materials, generally, the introduction of fillers brings a much more complex behavior (e.g. dielectric losses, interfacial effects), weaknesses (e.g. electrical strength, extra ageing routes, sensibility to water) and some improvements (erosion resistance under discharges, UV-protection). Nanocomposites have certainly positive effects in respect to microcomposites in the sense that they tend to maintain the advantages brought by filling the material and to attenuate drawbacks. Even though intrinsic properties are valuable, their development in this field is still dependent on reliability assessment, on processing constrains, availability and economical considerations.

\subsection{Concluding Remarks}

The purpose of this chapter was to present the main polymer ageing types likely to take place in practice and, in particular, to provide the reader with important pieces of information such as:

1 There is not a single ageing type, but several ageing types susceptible to superimpose and interact.

2 Ageing obeys a more or less complex set of physical and chemical mechanisms, each one obeying a well-known kinetic law.

3 In the past half century, researchers have made many efforts to elucidate these elementary mechanisms. Now, the main challenge is the elaboration of nonempirical kinetic models simulating satisfyingly the kinetics of such complex ageing processes (for instance, chemical ageings involving several dozens of chemical reactions, kinetically controlled by reactant diffusion, etc.) and their consequences on polymer structure, and physical and mechanical properties at the pertinent scales: the molecular, the macromolecular and the macroscopic scales;

4 Problems of thermal and radio-oxidation, and hydrolysis, kinetically controlled or not by diffusion reactant, are now solved nonempirically using numerical models derived from realistic mechanistic schemes (respectively, $[57,58]$ ). However, significant efforts remain to be made in the field of polymer photo-oxidation and interphase hydrolysis in composites materials, stacked assemblies, etc. The problem of interactions between oxidation and humid ageing is also interesting. Indeed, on the one hand, some oxidized groups can be highly polar (for instance, alcohols and acids). They can therefore modify substantially the water transport properties into a thick polymer piece. On the other hand, other oxidized groups (for instance, esters and amides) can hydrolyze easily.

5 Actual ageing phenomena depend on the environmental stresses acting on materials. Besides the skills in materials chemistry needed to apprehend the stability range of materials, there are fields like electrical ageing that are still unsatisfactorily explored when dealing with long-term ageing, particularly for multiphase and composite systems. 


\section{References}

1. Colin X., Audouin L., Verdu J., Polym. Deg. Stab. 92(5), 886-897, 898-905 and 906-914 (2007).

2. Colin X., Audouin L., Verdu J., Rozental M., Rabaud B., Martin F., Bourgine F., Polym. Eng. Sci. 49(7), 1429-1437 and 49(8), 1642-1652 (2009).

3. Struik L.C.E., Physical Ageing in Amorphous Polymers and Other Materials, Elsevier, Amsterdam, 1978.

4. Tiemblo P., Guzman J., Riande E., Mijangos C., Reinecke, H., Polymer 42, 4817-4823 (2001); ibid 8321.

5. Bernier G.A., Kambour P., Macromolecules 1, 393 (1968).

6. Mc Kague E.L., Halkias E., Reynolds J.D., J. Compos. Mater. 9, 2 (1975).

7. Shen C.H., Springer G.S., J. Compos. Mater. 10, 2 (1976).

8. Whitney J.-M., Browning C.E., ASTM STP 658, 33 (1978).

9. Müller-Plathe F., Polymerica Acta 45, 259-293 (1994).

10. Masaro L., Zhu X.X., Prog. Polym. Sci. 24, 731-775 (1999).

11. George S., Thomas S., Prog. Polym. Sci. 26, 985-1017 (2001).

12. Wypych G., Handbook of Plasticizers, ChemTec Publishing, Toronto, Canada, 151-170, 2004.

13. Wypych G., PVC Degradation and Stabilization, ChemTec Publishing, Toronto, Canada, 2008.

14. Saito O., J. Phys. Soc. Jap. 13(2), 198 (1958) and 13(12), 1451 (1958).

15. Fayolle B., L. Audouin, J. Verdu, Polym. Deg. Stab. 70(3) 333 (2000).

16. Bellenger V., Verdu J., J. Appl. Polym. Sci. 28, 2599-2609 and 2677-2688 (1983).

17. Bauer D.R., Mielewski D.F., Gerlock J.L., Polym. Deg. Stab. 38, 57-68 (1992).

18. Griffith A.A., Phil. Trans. Roy. Soc., Series A 221, 163-198 (1920).

19. Audouin L., Verdu J., in Radiation Effects on Polymers, R.L. Clough and S.W. Shalaby (Eds), ACS Symposium Series 475, American Chemical Society, Washington D.C., p. 473, 1991.

20. Bolland J.L., Gee G., Trans. Faraday Soc. 42(3-4), 236 and 244 (1946).

21. Kamiya Y., Niki E., in Aspects of Degradation and Stabilisation of Polymers, H.H.G. Jellinek (Ed.), Elsevier, New York, p. 86 (1978).

22. Korcek S., Chenier J.H.B., Howard J.A., Ingold K.U., Can. J. Chem. 50, 2285 (1972).

23. Coquillat M., Verdu J., Colin X., Audouin L., Nevière R., Polym. Deg. Stab. 92(7), 1334-1342 (2007).

24. Gillen K.T., Wise J., Clough R.L., Polym. Deg. Stab. 47, 149 (1995).

25. Colin X., Verdu J., Plastics Rubber Comp.: Macromol. Eng. 32(8/9), 349-356 (2003).

26. Assadi R., Colin X., Verdu, J., Polymer 45(13), 4403-4412 (2004).

27. Colin X., Richaud E., Verdu J., Monchy-Leroy C., Rad. Phys. Chem., 79(3), 365-370 (2010).

28. Verdu J., Rychly J., Audouin L., Polym. Deg. Stab. 79(3), 503 (2003).

29. Bordwell F.G., Zhang X.M.A.N., J. Phys. Org. Chem. 8(8), 529 (1995).

30. Mulder P., Saastad O.W., Griller D., J. Amer. Chem. Soc. 110(12), 4090 (1988).

31. Zhu Q., Zhang X.-M., Fry A.J., Polym. Deg. Stab. 57(1), 43 (1997).

32. Schwarzenbach K., Gilg B., Müller D., Knobloch G., in Plastic Additives Handbook, 5 th edition, H. Zweifel (Ed.), Hanser, Munich, Switzerland, 2000.

33. Shelton R.J., in Polymer Stabilization, W.L. Hawkins (Ed.), Wiley-Interscience, New -York, p. 104, 1971.

34. Hawkins W.L., Worthington M.A., J. Polym. Sci. 62(174), S106 (1962).

35. Kuzminskii A.S., Lyubchanskaya L., Khitrova N., Bass S., Rubber Chem. Technol. 26, 858 (1953).

36. Zweifel H., Plastics Additives Handbook, 5th edition, Hanser, Munich, Switzerland, 2000.

37. Hawkins W.L., Worthington M.A., J. Polym. Sci. Part A 1(11), 3489 (1963).

38. Pospisil J., Nespurek S., in Handbook of Polymer Degradation, S. Halim Hamid (Ed.), Marcel Dekker, New York, p. 259, 2000.

39. Teyssèdre G., Grimau M., Bernès A., Martinez J.J., Lacabanne C., Polymer 35, 4397-4403 (1994).

40. Teyssèdre G., Lacabanne C., Ferroelectrics 171, 125-144 (1995)

41. Boudet A., Teyssedre G., Caramaro L., Chabert B., Bull. Soc. Chim. Belg. 98, 747-750 (1989).

42. Gautier, L; Mortaigne, B; Bellenger, V., Composites Sci. Technol. 59(16), 2329-2337, 1999.

43. Fothergill J. C., IEEE Trans. Electr. Insul. 26, 1124-1129 (1991).

44. Kim H.K., Shi F.G., IEEE Trans. Dielectr. Electr. Insul. 8, 248-252 (2001). 
45. Morshuis, P.H.F., IEEE Trans. Dielectr. Electr. Insul. 12, 905-913 (2005).

46. Laurent C., Teyssedre G., Nucl. Instr. and Meth. in Phys. Res. B 208, 442-447 (2003).

47. Teyssedre G., Laurent C., IEEE Trans. Dielectr. Electr. Insul. 12, 857-875 (2005).

48. Starr W.T., IEEE Trans. Electr. Insul. 25, 125-136 (1990).

49. Englund V., Hjertberg T., Huuva R., Gubanski S.M., Proc. 2007 International Conference on Solid Dielectrics, Winchester, UK, July 8-13, 203-206 (2007).

50. Peruzzotti F., Martinotto L., Del Brenna M., in Cable, in particular for transport or distribution of electrical energy and insulating composition, US Patent 6696154, 2004.

51. Englund V., Huuva R., Gubanski S.M., Hjertberg T., Polymer Degr. Stab. 94, 823-833 (2009).

52. Sawa G., IEEE Trans. Electr. Insul. 21, 841-846 (1986).

53. Reynders J.P., Jandrell I.R., Reynders S.M., IEEE Trans. Dielectr. Electr. Insul. 6, 620-631 (1999).

54. Zou C., Fothergill J.C., Rowe S. W., IEEE Trans. Dielectr. Electr. Insul. 15, 106-117 (2008).

55. Adohi I.P., Guillermin C., Rain P., Rowe S.W., 2004 Annual Report Conference on Electrical Insulation and Dielectric Phenomena, 158-162 (2004).

56. Natsui M., Echigo Y., Tanaka T., Ohki Y., Maeno T., Proc. 2008 International Symposium on Electrical Insulating Materials, Sept. 7-11, 2008, Yokkaichi, Mie, Japan, pp. 376-379 (2008).

57. Colin X., Fayolle B., Audouin L., Verdu J., in Advanced in Chemistry Series 1004: Polymer Degradation and Performance, M.C. Celina, J.S. Wiggins and N.C. Billingham (Eds), Oxford University Press, Washington, pp 121-134, 2008.

58. Jacques B., Werth M., Merdas I., Thominette F., Verdu J., Polymer 43, 6439 (2002). 